\title{
On Lagrangian and vortex-surface fields for flows with Taylor-Green and Kida-Pelz initial conditions
}

\author{
YUE YANG† AND D. I. PULLIN \\ Graduate Aerospace Laboratories, 205-45, California Institute of Technology, \\ Pasadena, CA 91125, USA \\ (Received 16 February 2010; revised 31 May 2010; accepted 1 June 2010)
}

For a strictly inviscid barotropic flow with conservative body forces, the Helmholtz vorticity theorem shows that material or Lagrangian surfaces which are vortex surfaces at time $t=0$ remain so for $t>0$. In this study, a systematic methodology is developed for constructing smooth scalar fields $\phi(x, y, z, t=0)$ for Taylor-Green and KidaPelz velocity fields, which, at $t=0$, satisfy $\omega \cdot \nabla \phi=0$. We refer to such fields as vortex-surface fields. Then, for some constant $C$, iso-surfaces $\phi=C$ define vortex surfaces. It is shown that, given the vorticity, our definition of a vortex-surface field admits non-uniqueness, and this is presently resolved numerically using an optimization approach. Additionally, relations between vortex-surface fields and the classical Clebsch representation are discussed for flows with zero helicity. Equations describing the evolution of vortex-surface fields are then obtained for both inviscid and viscous incompressible flows. Both uniqueness and the distinction separating the evolution of vortex-surface fields and Lagrangian fields are discussed. By tracking $\phi$ as a Lagrangian field in slightly viscous flows, we show that the well-defined evolution of Lagrangian surfaces that are initially vortex surfaces can be a good approximation to vortex surfaces at later times prior to vortex reconnection. In the evolution of such Lagrangian fields, we observe that initially blob-like vortex surfaces are progressively stretched to sheet-like shapes so that neighbouring portions approach each other, with subsequent rolling up of structures near the interface, which reveals more information on dynamics than the iso-surfaces of vorticity magnitude. The non-local geometry in the evolution is quantified by two differential geometry properties. Rolled-up local shapes are found in the Lagrangian structures that were initially vortex surfaces close to the time of vortex reconnection. It is hypothesized that this is related to the formation of the very high vorticity regions.

Key words: topological fluid dynamics, turbulence theory, vortex dynamics

\section{Introduction}

The paradigm of vortex dynamics has become useful within theoretical fluid mechanics with a substantial impact on many applications in fluid systems (e.g. Saffman 1992 and Majda \& Bertozzi 2001). Helmholtz (1858) obtained a central result in vortex dynamics, namely that vortex lines or surfaces move with fluid in a perfectly inviscid flow. This means that material lines or surfaces that were initially 
vortex lines or surfaces are vortex lines or surfaces for all time. Presently we define a 'vortex surface' (the term 'vorticity surface' is used instead in some literature), examples of which are tube-like or sheet-like geometries, as a smooth surface or manifold embedded within a three-dimensional velocity field, which, at a time instant $t$, has the property that the local vorticity vector is tangent at every point on the surface. We further define a 'Lagrangian field', say $\phi(x, y, z, t)$, as a scalar field whose iso-surfaces evolve as Lagrangian material surfaces and a 'vortex-surface field' as a smooth scalar field whose iso-surfaces are vortex surfaces, either instantaneously or in evolution. This concept was utilized by Clebsch (1859) in developing a Lagrangian formulation of the Euler equations for an incompressible inviscid flow where each vortex line is expressed, at least locally, by the intersection of iso-surfaces of Clebsch potentials. More recently, the mathematical formulation of vortex dynamics has been interpreted in terms of the Clebsch potentials (e.g. Lamb 1932 and Pumir, Shraiman \& Siggia 1992) while the classical Clebsch representation has been extended to build Eulerian-Lagrangian frameworks for Navier-Stokes dynamics (e.g. Constantin 2001, Ohkitani \& Constantin 2003 and Cartes, Bustamante \& Brachet 2007). The global existence of vortex-surface fields and smooth Clebsch potentials is, however, less obvious. Although the Helmholtz vorticity theorem has been utilized for more than 150 years, we know of no Lagrangian study on the evolution of complete and globally smooth vortex surfaces in general three-dimensional flows. Challenges exist in both the initial construction and tracking of vortex surfaces or vortex-surface fields.

In dynamical systems theory, the construction of vortex surfaces from a given velocity field is related to finding two-dimensional invariant manifolds of a threedimensional vector field. Although, in general, global manifolds cannot be found analytically, there are several numerical methods under development for computing a manifold of a vector field (see Krauskopf et al. 2005). In the present study, we find that flows that begin from Taylor-Green (referred to as TG below) and KidaPelz (referred to as KP below) initial conditions are good candidates for the study of vortex surfaces, because their initial topology is relatively simple and both of these periodic flows have many symmetries to facilitate construction of initial vortex surfaces. In particular, we will develop a high-accuracy method for finding spacefilling, vortex-surface fields for these two defined velocity fields. At the same time, the recent development of tracking methodologies for finite-sized Lagrangian structures (e.g. Lindsay \& Krasny 2001, Goto \& Kida 2007 and Yang, Pullin \& Bermejo-Moreno 2010) offer effective approaches to numerical simulation of the evolution of vortex surfaces in a high-Reynolds-number flow or even an inviscid flow with the aid of substantial computational resources.

In general, a vortex surface will be persistently stretched in inviscid and perhaps high-Reynolds-number flows, which causes the growth of vorticity. This mechanism is crucial for the cascade process and dissipation distribution in turbulence. To express them in mathematical form, Taylor \& Green (1937) proposed a divergence-free velocity field with Fourier basis functions, as the initial condition of the NavierStokes equations to show that, when the Reynolds number is high, the mean-squared vorticity increased during the early stage of the flow evolution and reached a maximum after a finite time. Subsequently, the so-called TG flow was investigated by Brachet et al. (1983) using numerical simulations. From this simple sinusoidal vector field with certain reflectional and rotational symmetries, Brachet et al. (1983) found that a TG flow can develop characteristics of Kolmogorov statistics of turbulence at a later stage in a high-Reynolds-number flow. Another typical flow with simple initial conditions, the KP flow, was introduced by Kida (1985), who showed that it contains more 
symmetries than the TG flow. Flows with KP initial conditions have been studied in both viscous and inviscid cases (e.g. Boratav \& Pelz 1994 and Pelz 2001).

The topology and geometry in evolution of vortex-surface fields and vortex surfaces are important for many aspects in theoretical fluid mechanics. From the Helmholtz theorem, for a strictly inviscid flow, a vortex surface is a Lagrangian structure (material surface), which is topologically invariant in time provided that the velocity field remains smooth. The nature of solutions of the three-dimensional Euler equations remains a challenging open problem (e.g. Majda \& Bertozzi 2001 and Kerr 1993). Some studies showed that nearly singular, sheet-like vortex structures may deplete the growth of nonlinearity so as to prevent finite-time singularity formation in Euler flows (e.g. Hou \& Li 2008). Constantin, Majda \& Tabak (1994) introduced a twodimensional analogy using a scalar gradient as the analogue of vorticity and level sets of the scalar field as the analogue of vortex lines. Their analysis showed that the flow in the vicinity of hyperbolic saddles is highly nonlinear and potentially singular. Describing the evolution of vortex surfaces in a general three-dimensional flow, however, appears to pose difficulties. It is expected that the topology of vortex surfaces will change when the Reynolds number is finite owing to vortex reconnection, for example in the collapse of two vortex tubes under excessive straining (see Kida \& Takaoka 1994). Subsequent roll-up of vortex sheets induced by Kelvin-Helmholtz instability may provide an agent of transition from a laminar flow to a turbulent flow. In these scenarios, knowledge of the geometry of vortex surfaces may help us to construct vortex models of the inertial range and fine-scale range of high-Reynoldsnumber turbulence (Pullin \& Saffman 1998).

Unlike the velocity, which can be rapidly redistributed over all space by the pressure field, an initially localized distribution of vorticity tends to remain localized, which implies that the vorticity field may contain persistent structures. An agreed-upon definition for vortical structures, however, remains elusive. Iso-surfaces of vorticity magnitude provides one method for visualizing instantaneous vortical structures, but this may be inadequate, in particular, for detecting coherent structures in wallbounded and homogeneous shear flows (Jeong \& Hussain 1995). Hence, several popular criteria to identify vortical structures are presently available, most based on invariants or eigenvalues of the local velocity gradient tensor, for example the $Q$ criterion (Hunt, Wray \& Moin 1988), the $\Delta$-criterion (Chong, Perry \& Cantwell 1990) and the $\lambda_{2}$-criterion (Jeong \& Hussain 1995). Similarities and differences between these criteria were discussed in Chakraborty, Balachandar \& Adrian (2005). An alternative to these Eulerian measures is provided by the frame-independent criterion (Haller 2005), which, in a Lagrangian sense, appears to be capable of exposing detailed vortical structures. According to the Helmholtz theorem, a primitive approach based on the identification of vortex surfaces has a clear physical meaning and unique topological and geometrical interpretation. It will, however, be seen that for a given vorticity field, the set of vortex surfaces is not unique. Nonetheless, observation of the evolution of three-dimensional vortex surfaces might be helpful in clarifying some aspects of vortex identification.

In this paper, we begin in $\S 2$ by introducing a systematic methodology for constructing vortex-surface fields for both TG and KP initial velocity fields. Equations describing the evolution of vortex-surface fields are then obtained in $\S 3$, and their existence and uniqueness properties are discussed for both inviscid and viscous incompressible flows. In $\S 4$, we review the numerical methods used presently for tracking Lagrangian structures, including the pseudo-spectral method for the Eulerian velocity field and the backward-particle-tracking method for the Lagrangian field. 
The growth of the divergence of evolving Lagrangian fields away from their vortexsurface-field initial conditions in a high-Reynolds-number viscous flow is studied and quantified in $\S 5$. Finally, we investigate the non-local and local geometries of approximate vortex surfaces in time evolution. Some conclusions are drawn in $\S 6$.

\section{Construction of vortex-surface fields}

\subsection{Partial-differential-equation formulation}

We develop a methodology for construction of vortex-surface fields for some simple given velocity fields. A discussion of their evolution in time and their relation to Lagrangian fields is postponed until $\S 3$. A vortex line is defined by

$$
\frac{\mathrm{d} \boldsymbol{X}}{\mathrm{d} s}=\frac{\boldsymbol{\omega}}{|\boldsymbol{\omega}|},
$$

where $s$ is the arclength along the vortex line and the vorticity is $\boldsymbol{\omega} \equiv \nabla \times \boldsymbol{u}$, with the Eulerian velocity $\boldsymbol{u}$. For the given vorticity field, we now assume the existence of a smooth, space-filling scalar function $\phi(x)$ that has the property that the vorticity vector is tangent to iso-surfaces of $\phi(\boldsymbol{x})$ everywhere except perhaps at a finite number of critical points. It follows that

$$
\frac{\mathrm{d} \phi}{\mathrm{d} s}=\frac{\mathrm{d} \phi}{\mathrm{d} \boldsymbol{X}} \frac{\mathrm{d} \boldsymbol{X}}{\mathrm{d} s}=0
$$

Substituting (2.1) into (2.2) yields the constraint for $\phi$ :

$$
\omega \cdot \nabla \phi=\omega_{x}(x, y, z) \frac{\partial \phi}{\partial x}+\omega_{y}(x, y, z) \frac{\partial \phi}{\partial y}+\omega_{z}(x, y, z) \frac{\partial \phi}{\partial z}=0 .
$$

We will take (2.3) as our fundamental definition of a vortex-surface field. For given $\boldsymbol{\omega}$, we can view this as a homogeneous first-order partial differential equation (PDE) for $\phi$. We will see that (2.3) does not admit unique solutions, but it may admit useful solutions. Equation (2.3) is similar to the PDE formulation proposed by Dieci, Lorenz \& Russell (1991) for computing numerical approximations to invariant tori corresponding to ordinary differential equations (ODEs) like (2.1). An alternative form of this PDE formulation is to find a two-dimensional invariant manifold in a three-dimensional real space with a local parametrization $(x, y, g(x, y))$. Then the graph of $g(x, y)$ should be tangential to a given vector field $f(x, y, g(x, y))$ as

$$
\left[\frac{\partial}{\partial x} g(x, y), \frac{\partial}{\partial y} g(x, y),-1\right] \cdot f(x, y, g(x, y))=0 .
$$

The major difference between our proposed relation (2.3) and (2.4) is that, from (2.3), we seek a set of invariant manifolds as space-filling iso-surfaces of $\phi$ within the whole domain of some particular vector field, while only an individual invariant manifold can be found from (2.4).

Equation (2.4) was solved by finite-difference methods and Newton's iteration in Dieci et al. (1991), while Mingyu, Küpper \& Masbaum (1997) showed that a Fourier method is more accurate for computing invariant tori of a vector field with periodic boundary conditions. To find a $\phi$ satisfying (2.3) in the present study, at an initial time for a class of simple vorticity fields with periodic boundary conditions, we expand $\omega$ 
and $\phi$ as Fourier series:

$$
\begin{aligned}
\omega_{\alpha} & =\sum_{k_{1}, k_{2}, k_{3}} \Omega_{k_{1}, k_{2}, k_{3}}^{\alpha} \mathrm{e}^{\mathrm{i} k_{\beta} x_{\beta}}, \quad \alpha=1,2,3, \quad \beta=1,2,3, \\
\phi & =\sum_{k_{1}, k_{2}, k_{3}} \Phi_{k_{1}, k_{2}, k_{3}} \mathrm{e}^{\mathrm{i} k_{\beta} x_{\beta}}, \quad \beta=1,2,3,
\end{aligned}
$$

where $\Omega_{k_{1}, k_{2}, k_{3}}^{\alpha}$ are given by the vorticity, $\Phi_{k_{1}, k_{2}, k_{3}}$ are to be determined and $k_{\beta} x_{\beta}$ denotes the summation convention over $\beta$. Substituting both Fourier expansions into (2.3) and then collecting coefficients of each Fourier mode by symbolic manipulation yield

$$
\sum_{k_{1}, k_{2}, k_{3}} \Pi_{k_{1}, k_{2}, k_{3}} \mathrm{e}^{\mathrm{i} k_{\beta} x_{\beta}}=0,
$$

where $\Pi_{k_{1}, k_{2}, k_{3}}$ is a linear polynomial in terms of unknown $\Phi_{k_{1}, k_{2}, k_{3}}$. Substituting all $\Pi_{k_{1}, k_{2}, k_{3}}=0$ into (2.7), we obtain a system of homogenous linear equations

$$
\boldsymbol{C \Phi}=0,
$$

where $\boldsymbol{C}$ is the $m_{c} \times n_{c}$ coefficient matrix for $\Phi_{k_{1}, k_{2}, k_{3}}$ with rank $r_{c}$, and $\boldsymbol{\Phi}$ is a suitably constructed Fourier coefficient vector in (2.6). If there exists a vortex-surface field $\phi(\boldsymbol{x})$ exactly satisfying the relation (2.3), the corresponding homogeneous system (2.8) with $r_{c}<n_{c}$ must have $n_{c}-r_{c}$ non-trivial solutions. Otherwise, if $r_{c}=n_{c}$, we have to seek an approximate solution of (2.8). We now show how to construct such initial vortex-surface fields for three typical simple flows using this methodology.

\subsection{Taylor-Green flow}

The generalized initial condition $\boldsymbol{u}(x, y, z, t=0)$ of the TG flow is given by, up to an arbitrary real multiplicative constant of $\theta$ (see Brachet et al. 1983),

$$
\left.\begin{array}{l}
u_{x}=\frac{2}{\sqrt{3}} \sin \left(\theta+\frac{2 \pi}{3}\right) \sin x \cos y \cos z, \\
u_{y}=\frac{2}{\sqrt{3}} \sin \left(\theta-\frac{2 \pi}{3}\right) \cos x \sin y \cos z, \\
u_{z}=\frac{2}{\sqrt{3}} \sin \theta \cos x \cos y \sin z .
\end{array}\right\}
$$

The corresponding vorticity is

$$
\left.\begin{array}{l}
\omega_{x}=-(\sqrt{3} \sin \theta+\cos \theta) \cos x \sin y \sin z, \\
\omega_{y}=(\sqrt{3} \sin \theta-\cos \theta) \sin x \cos y \sin z, \\
\omega_{z}=2 \cos \theta \sin x \sin y \cos z .
\end{array}\right\}
$$

Based on the mirror and rotational symmetries in the TG flow, the region $0 \leqslant x, y, z \leqslant 2 \pi$ is conventionally termed the 'periodicity box'; the region $0 \leqslant x, y, z \leqslant \pi$ is termed the 'impermeable box'; and the region $0 \leqslant x, y, z \leqslant \pi / 2$ is termed the 'fundamental box' (see Brachet et al. 1983).

From (2.10), the wavenumbers for the Fourier expansion of $\omega_{\alpha}$ are \pm 1 in the TG flow at $t=0$. Assume that the corresponding $\phi$ has the same Fourier modes, namely

$$
\phi=\sum_{k_{1}, k_{2}, k_{3}= \pm 1} \Phi_{k_{1}, k_{2}, k_{3}} \mathrm{e}^{\mathrm{i} \mathrm{k}_{\beta} x_{\beta}} .
$$



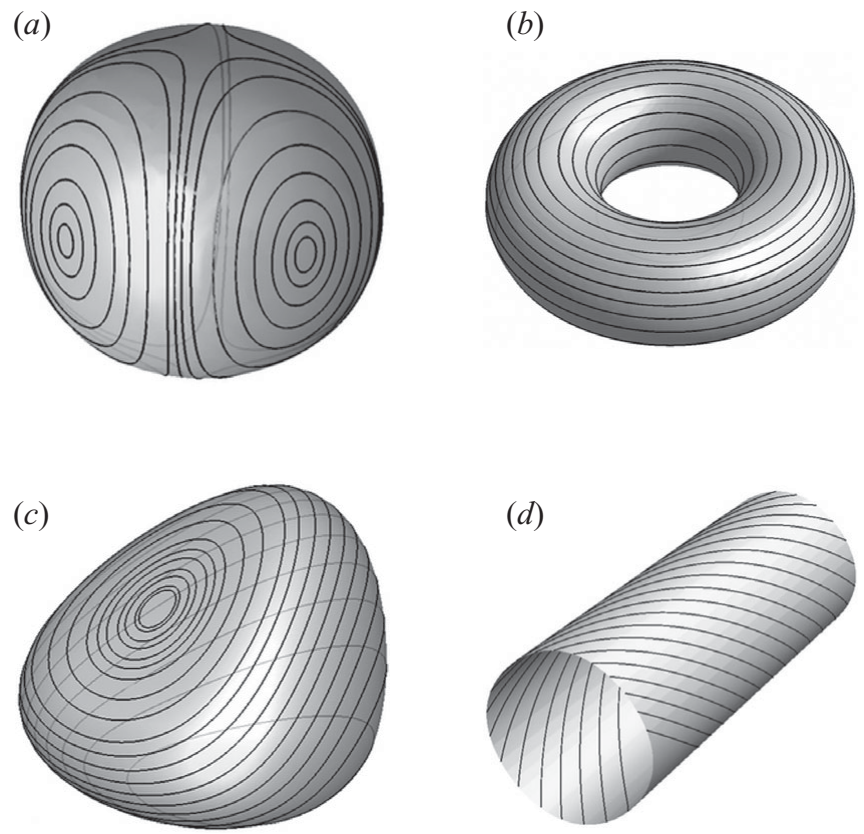

FIgURE 1. Typical vortex surfaces and vortex lines in simple flows at the initial time. (a) TG flow, $(b)$ vortex ring, $(c) \mathrm{KP}$ flow and $(d) \mathrm{ABC}$ flow (integrable).

The expansion (2.5) for the vorticity is obtained from (2.10) by symbolic manipulation. Substituting (2.5) and (2.11) into (2.3) and then setting all the Fourier coefficients in (2.7) equal to zero leads to an $18 \times 8$ coefficient matrix $\boldsymbol{C}$, with $r_{c}=7$ in (2.8). Thus, we can obtain a non-trivial fundamental solution $(1,1,1,1,1,1,1,1)^{\mathrm{T}}$. If we let $\Phi_{1,1,1}=1 / 8$ in (2.11), then we have the solution

$$
\phi_{T G}=\cos x \cos y \cos z,
$$

and every iso-surface of $\phi_{T G}$ is a vortex surface for the TG flow with an arbitrary $\theta$ at the initial time. An example of a vortex surface as an iso-surface of (2.12) is shown in figure 1( $a$ ). All the vortex lines computed by (2.1) and (2.10) with $\theta=0$ lie on the surface. The special case with $\theta=0$ in (2.9) was discussed at length in Brachet et al. (1983), and this will be used for all computations in this paper.

We note that (2.12) is not unique. Since $\phi_{T G}$ satisfies (2.3), by the chain rule, so does any function $Q\left(\phi_{T G}\right)$. If $\phi_{T G} \rightarrow Q$ is $1-1$, this is just a relabelling so that iso-surfaces $\phi_{T G}(x, y, z)=C$ and $Q(x, y, z)=Q(C)$ are identical geometrically. But for a given vorticity field, it appears that vortex lines can be bundled or arranged on smooth surfaces in infinitely many different ways. Hence, we cannot rule out solutions to (2.3) that are continuous scalar fields not equivalent to either a relabelling of $\phi_{T G}$ or to a mapping under the symmetries of the TG flow, and whose iso-surfaces could therefore have a different topology or geometry. The existence of additional classes of vortex-surface fields that are not connected by relabelling mappings may enable the construction of non-trivial Clebsch potentials (e.g. Lamb 1932). This issue is revisited in $\S 2.5$.

In some special cases such as $\theta= \pm \pi / 6, \pm \pi / 2, \pm 5 \pi / 6$ in (2.9), one vorticity component in (2.10) is zero. For example, as $\theta= \pm \pi / 2, \omega_{z}=0$, the constraint (2.3) is 
reduced to

$$
\omega_{x}(x, y, z) \frac{\partial \phi}{\partial x}+\omega_{y}(x, y, z) \frac{\partial \phi}{\partial y}=0 .
$$

Therefore, as long as $\phi(x, y)$ satisfies $(2.13), \phi(x, y) f(z)$ can be the initial vortex-surface field satisfying (2.3). Here, $f(z)$ is an arbitrary function, which implies that iso-surfaces of this vortex-surface field have a non-unique topology. From our methodology, two fundamental solutions, $\cos x \cos y \exp ( \pm \mathrm{i} z)$, can be obtained from (2.8) for this case. The real part of this solution is equivalent to (2.12). Thus, presently we will take (2.12) as our basic vortex-surface field solution of (2.3) for the TG initial vorticity field with $\theta=0$. The issue of non-uniqueness for both the definition of $\phi$ and its evolution will be revisited subsequently.

In addition, it is obvious that for a given three-dimensional vorticity field with only one non-trivial component, the vortex-surface field must exist and its topology is non-unique. For example, in cylindrical coordinates $(r, \theta, z)$, consider the initial vorticity field of a vortex ring centred at $(0,0,0)$ with radius $R$ and vorticity $\omega_{V R}=\left(0, \exp \left(-\left((r-R)^{2}+z^{2}\right), 0\right)\right)$. Any regular scalar field $\phi(r, z)$ can satisfy $\omega_{V R} \cdot \nabla \phi=0$, such as $\phi=\exp \left(-\left((r-R)^{2}+z^{2}\right)\right)$, with the topology of the torus in figure $1(b)$ or $\phi=\exp \left(-\left(r^{2}+z^{2}\right)\right)$, with the topology of the sphere.

\subsection{Kida-Pelz flow}

The TG flow is a special case for which we can find a simple scalar field (2.12) satisfying the constraint (2.3). For other vorticity fields with more Fourier modes, for example the KP flow, we are unable to find a simple exact solution, because it appears that $r_{c}=n_{c}$ in (2.8) with a finite set of Fourier modes. The initial condition of the KP flow is defined as

$$
\left.\begin{array}{l}
u_{x}=\sin x(\cos 3 y \cos z-\cos y \cos 3 z) \\
u_{y}=\sin y(\cos 3 z \cos x-\cos z \cos 3 x) \\
u_{z}=\sin z(\cos 3 x \cos y-\cos x \cos 3 y)
\end{array}\right\}
$$

and the corresponding vorticity is

$$
\left.\begin{array}{l}
\omega_{x}=-2 \cos 3 x \sin y \sin z+3 \cos x(\sin 3 y \sin z+\sin y \sin 3 z), \\
\omega_{y}=-2 \cos 3 y \sin z \sin x+3 \cos y(\sin 3 z \sin x+\sin z \sin 3 x), \\
\omega_{z}=-2 \cos 3 z \sin x \sin y+3 \cos z(\sin 3 x \sin y+\sin x \sin 3 y)
\end{array}\right\}
$$

We now develop an optimization methodology for obtaining $\phi_{K} P$ that approximately satisfies (2.3) for the KP flow at the initial time. We note that the scale of this problem mainly depends on the number of unknown coefficients in (2.8),

$$
n_{c}=N_{F}=\left(2 K_{F}+1\right)^{3} \text {, }
$$

where $K_{F}$ is the maximum wavenumber in (2.6). The cubic growth of $N_{F}$ with increasing $K_{F}$ in the Fourier expansion generally makes the convergence rate very slow. The scale of this problem solved by a direct method used for the TG initial condition is thus unaffordable for the KP case.

Equation (2.15) implies that vortex lines in the KP flow have reflectional and octahedral symmetries (see Kida 1985 and Pelz 2001). In particular, as shown in figure 2, we find a family of closed and unknotted vortex lines that is confined within a fundamental tetrahedron with vertices at, e.g. $(0,0,0),(-\pi / 2, \pi / 2,-\pi / 2)$, $(-\pi / 2,-\pi / 2,-\pi / 2)$ and $(0,0,-\pi / 2)$. Four fundamental tetrahedrons with rotational symmetries can constitute a pyramid drawn in solid thick lines in figure 2 . The box drawn in dotted lines lies in $-\pi / 2 \leqslant x, y, z \leqslant \pi / 2$. It comprises six pyramids 


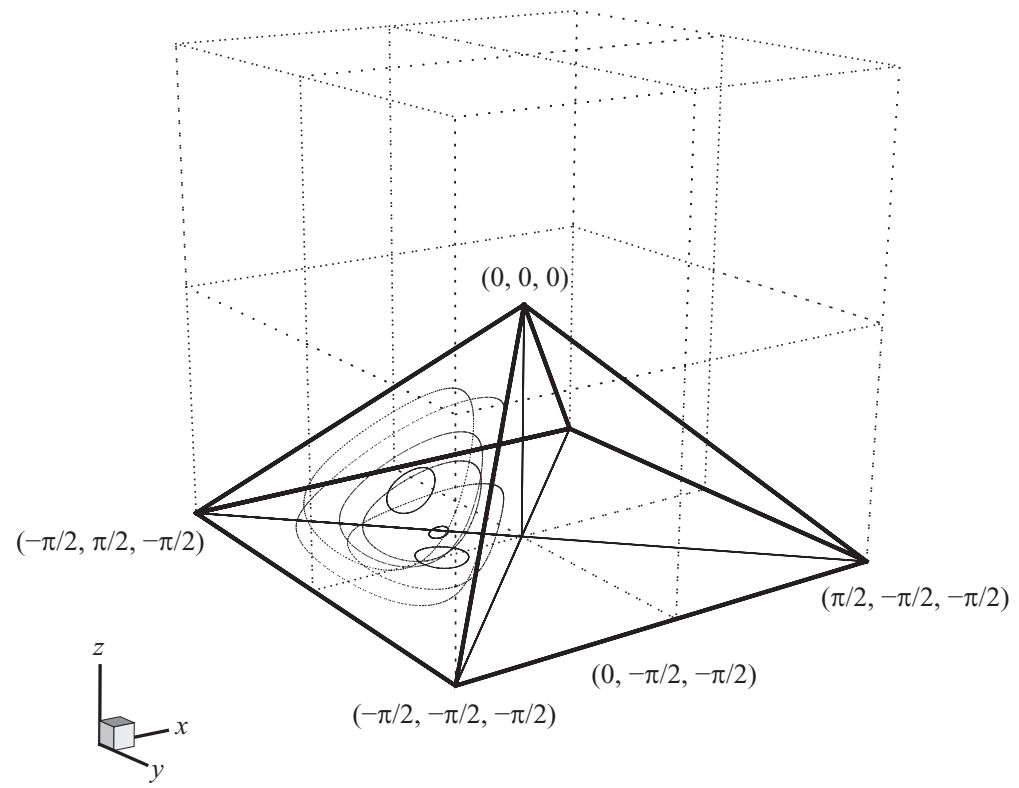

FIgURE 2. Symmetries of vortex lines in the KP flow. Some vortex lines are integrated from random points in a fundamental tetrahedron. The dotted box lies in $-\pi / 2 \leqslant x, y, z \leqslant \pi / 2$.

with rotational and reflectional symmetries. Finally, the box with periodic boundary conditions lies in $-\pi / 2 \leqslant x, y, z \leqslant 3 \pi / 2$, consisting of eight such boxes in dotted lines with additional rotational and reflectional symmetries. This box with side $2 \pi$ thus has 192 fundamental tetrahedrons. These symmetries suggest the construction of new orthogonal basis functions $\widetilde{\psi}_{i}$ to obtain faster convergence. Presently, the development of $\widetilde{\psi}_{i}$ utilizes a tailored numerical scheme, described in detail in Appendix A. A sparse representation, $\phi_{K P}$, can then be expanded in these basis functions as

$$
\phi_{K P}=\sum_{i=1}^{N_{\psi}} \Psi_{i} \widetilde{\psi}_{i}
$$

The expansion (2.17) can be converted to Fourier space by symbolic manipulations,

$$
\phi_{K P}=\sum_{k_{1}, k_{2}, k_{3}}^{K_{F}} \Phi_{k_{1}, k_{2}, k_{3}}^{K P} \mathrm{e}^{\mathrm{i} k_{\beta} x_{\beta}}, \quad \beta=1,2,3,
$$

where $\Phi_{k_{1}, k_{2}, k_{3}}^{K P}$ is a linear polynomial in terms of the unknown coefficient $\Psi_{i}$ in (2.17) and $K_{F}$ is the maximum wavenumber.

Substituting (2.17) and (2.5) with the vorticity (2.15) into (2.3), we can obtain an expansion as in (2.7). Note that $\Pi_{k_{1}, k_{2}, k_{3}}$ is a linear polynomial in terms of the coefficient $\Psi_{i}$ of the basis function $\widetilde{\psi}_{i}$ rather than $\Phi_{k_{1}, k_{2}, k_{3}}^{K P}$ in the Fourier expansion (2.18). This leads to a significantly faster convergence rate for obtaining an accurate approximate solution. From Appendix A, the number of coefficients for $\widetilde{\psi}_{i}$ with the same $K_{F}$ in $(2.18)$ is

$$
N_{\psi}=\left(K_{F}^{4}+24 K_{F}^{2}-112\right) / 2048 .
$$

Compared with the equivalent Fourier coefficient number (2.16), we can find that when $K_{F}$ is not too large, $N_{\psi} \ll N_{F}$ for the same resolution expressed by $K_{F}$. 


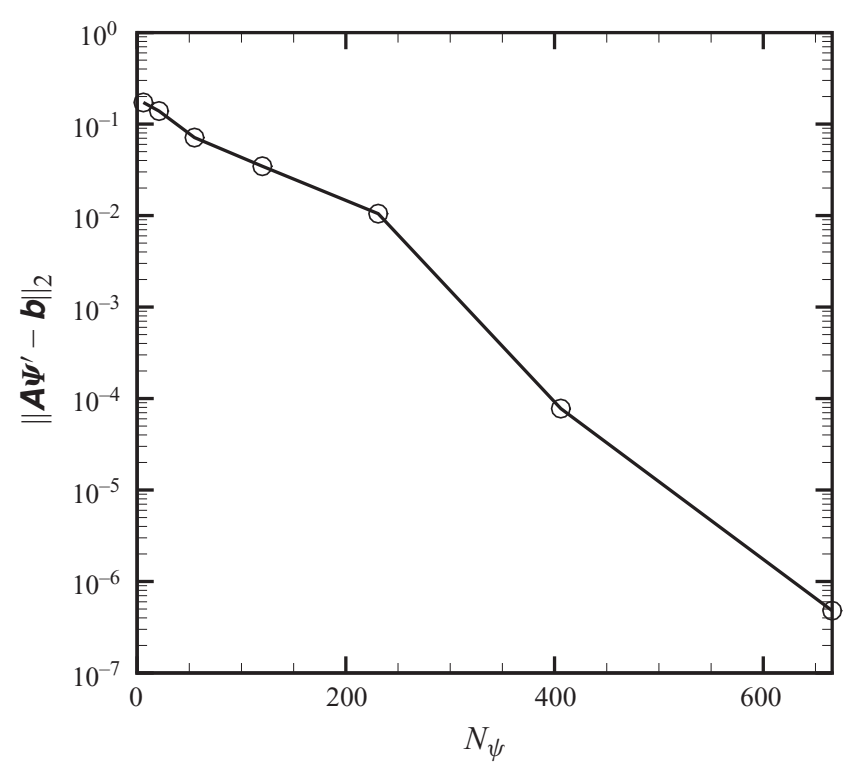

FIGURE 3. Convergence rate with $N_{\psi}$ for the approximate solution of the initial scalar field in the KP flow $\phi_{K P}$, where $N_{\psi}$ is computed from $K_{\psi}=3,5, \ldots, 17$ by (A 4) and (A 8).

Setting $\Pi_{k_{1}, k_{2}, k_{3}}=0$ in (2.7) with $\Psi_{i}$, we have a homogenous linear system

$$
\boldsymbol{C} \boldsymbol{\Psi}=0
$$

where $\boldsymbol{\Psi}$ is the corresponding coefficient vector in (2.17). The overdetermined system (2.20) with a finite number of coefficients appears to have only a trivial solution. To obtain a non-trivial coefficient vector $\boldsymbol{\Psi}$, we let $\Psi_{1}=1$ in $(2.17)$, which is equivalent to assuming the full octahedral symmetry on large-scale structures in the approximate solution, noting that $\widetilde{\psi}_{1}$ (see Appendix A) is proportional to

$$
(\cos 3 x \cos y-\cos x \cos 3 y)(\cos 3 y \cos z-\cos y \cos 3 z)(\cos 3 z \cos x-\cos z \cos 3 x) \text {. }
$$

Let $\boldsymbol{C}=\left(\begin{array}{ll}-\boldsymbol{b} & \boldsymbol{A}\end{array}\right)$ and $\boldsymbol{\Psi}=\left(\begin{array}{ll}\Psi_{1} & \boldsymbol{\Psi}^{\prime}\end{array}\right)^{\mathrm{T}}$. Then we can obtain a non-homogeneous overdetermined system:

$$
\boldsymbol{A} \boldsymbol{\Psi}^{\prime}=\boldsymbol{b} .
$$

This system may have a least-squares solution by

$$
\min _{\boldsymbol{\Psi}^{\prime}}\left\|\boldsymbol{A} \boldsymbol{\Psi}^{\prime}-\boldsymbol{b}\right\|_{2}^{2}
$$

where $\|\cdot\|_{2}$ denotes the $L^{2}$ norm. As shown in figure 3 , the residual $\left\|\boldsymbol{A} \boldsymbol{\Psi}^{\prime}-\boldsymbol{b}\right\|_{2}$ decreases exponentially with increasing $N_{\psi}$, which shows a strong convergence rate. Satisfactory accuracy could be obtained with $N_{\psi}=666$ with the corresponding $K_{F}=34$, while in the full Fourier expansion (2.6), the equivalent unknown coefficient number $N_{F}=328509$. This shows a significant scale reduction in this optimization problem using the proposed basis function $\widetilde{\psi}_{i}$. Further improvement on the smoothness of solutions from (2.22) is given in Appendix B.

Finally, $\phi_{K P}$ was obtained from (2.18) by the inverse fast Fourier transform. The volume-averaged $\left|\nabla \phi_{K P} \cdot \omega\right|$ is very small as $O\left(10^{-7}\right)$ and $\left|\phi_{K P}\right|_{\text {max }} \approx 0.648$, which shows that $\phi_{K P}$ obtained by the optimization methodology is an accurate non-trivial 


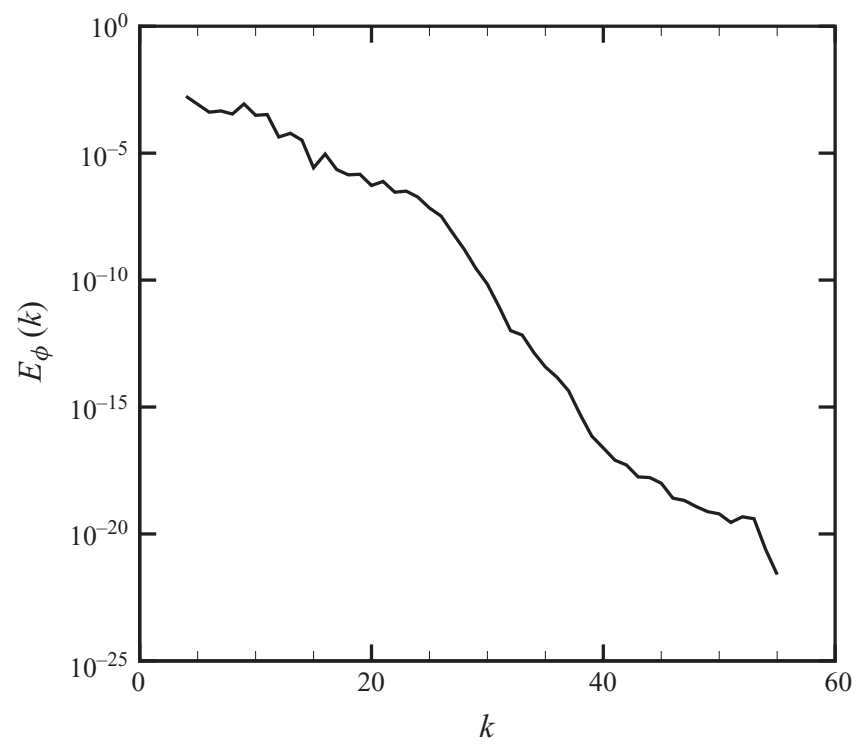

FIGURE 4. The spectrum of $\phi_{K P}$.

solution for (2.3). The spectrum of $\phi_{K P}$ in figure 4 also illustrates the good spectral convergence of this solution. An iso-surface of $\phi_{K P}$ in a fundamental tetrahedron with vortex lines on it is shown in figure $1(c)$, which can represent a vortex surface in the KP flow at the initial time. The code for the methodology in $\$ \S 2.2$ and 2.3 was written in Mathematica (Wolfram Research 2008) for symbolic manipulations and Fortran 90 for matrix computations. Furthermore, the optimization methodology in this section can also be utilized for the TG initial condition to uniquely determine the vortex-surface field (2.12).

\subsection{Arnold-Beltrami-Childress flow}

Another well-known simple flow is the Arnold-Beltrami-Childress (ABC) flow. The velocity field is

$$
\left.\begin{array}{l}
u_{x}=A \sin z+C \cos y, \\
u_{y}=B \sin x+A \cos z, \\
u_{z}=C \sin y+B \cos x .
\end{array}\right\}
$$

The streamlines, which are equivalent to vortex lines, in the ABC flow are chaotic for most values of the parameters $A, B$ and $C$ (Dombre et al. 1986). For the integrable case, where

$$
\boldsymbol{u}=(A \sin z, B \sin x+A \cos z, B \cos x),
$$

we can find a scalar field $\phi_{A B C}=B \sin x+A \cos z$ to satisfy the constraint (2.3). The iso-surface of $\phi_{A B C}$ corresponds to a simple vortex tube, as shown in figure $1(d)$. However, even for nearly integrable cases, the vortex lines become chaotic so that it appears to be difficult to find such a scalar field exactly satisfying (2.3), and in fact no such $\phi$ may exist. Moreover, we note that the ABC flow is stationary, so we cannot study the dynamics of vortex surfaces in this flow. The general problem of the existence of some non-trivial $\phi$ satisfying (2.3) for an arbitrary, spatially periodic vorticity field of the type encountered at a time instant in either forced or decaying homogeneous (box) turbulence remains, to the best of our knowledge, an open problem. 


\subsection{Vortex-surface fields and Clebsch potentials}

Vortex-surface fields are also helpful in representing the full Eulerian velocity field in terms of Lagrangian variables. Clebsch (1859) established an appealing Hamiltonian formulation as a compromise between Eulerian and Lagrangian descriptions of fluid dynamics (see also Lamb 1932 and Salmon 1988). In the Clebsch representation, the incompressible fluid velocity at any time instant can be expressed locally in terms of three scalar fields as

$$
\boldsymbol{u}(\boldsymbol{x})=\varphi_{1} \nabla \varphi_{2}-\nabla \varphi_{3},
$$

where $\varphi_{1}(\boldsymbol{x})$ and $\varphi_{2}(\boldsymbol{x})$, which can evolve as Lagrangian fields, are often called Clebsch potentials, and $\varphi_{3}$ is used for the solenoidal projection. Sometimes $\varphi_{1} \nabla \varphi_{2}$ is referred to as the 'impulse' variable (e.g. Russo \& Smereka 1999). It follows that the vorticity can be expressed by

$$
\boldsymbol{\omega}(\boldsymbol{x})=\nabla \varphi_{1} \times \nabla \varphi_{2},
$$

with the interesting geometric interpretation that vortex lines are the intersection of iso-surfaces of $\varphi_{1}$ and $\varphi_{2}$.

The global existence of the Clebsch potentials is, however, restricted to inviscid flows with vanishing helicity $H \equiv \int \boldsymbol{u} \cdot \boldsymbol{\omega} \mathrm{d} \boldsymbol{x}$ (see Salmon 1988). When $H=0$, e.g. in inviscid TG and KP flows, it is easy to verify from (2.27) that non-trivial $\varphi_{1}, \varphi_{2}$ and $\varphi_{3}$ satisfy the constraint for the vortex-surface field (2.3). This suggests that we can construct explicit Clebsch potentials from multiple independent vortex-surface fields, if the latter exist, for a given velocity field. We note that, when $H \neq 0$, it is still possible to find vortex-surface fields in some particular flows, such as the integrable $\mathrm{ABC}$ flow.

For the TG initial velocity field, we seek $n>1$ independent vortex-surface fields of the form

$$
\phi^{(i)}=A_{i} \cos ^{a_{i}} x \cos ^{b_{i}} y \cos ^{c_{i}} z, \quad i=1,2, \ldots, n,
$$

where $a_{i}, b_{i}$ and $c_{i}$ should be integers larger than or equal to zero for smooth and real vortex-surface fields and satisfy, using (2.3) and (2.10),

$$
\left(a_{i}+b_{i}-2 c_{i}\right) \cos \theta+\sqrt{3}\left(a_{i}-b_{i}\right) \sin \theta=0 .
$$

Hence, the geometry of vortex lines in figure $1(a)$ can be expressed by the intersections of iso-surfaces of two independent vortex-surface fields, as shown in figure 5.

Any three vortex-surface fields cannot be guaranteed to satisfy the Clebsch representation (2.26). We therefore take $\varphi_{1}=\phi^{(1)}$ and $\varphi_{2}=\phi^{(2)}$. Then, from (2.26), (2.27) and (2.29), we can find families of independent solutions for the Clebsch representation of the general TG initial field (2.9) of the form

$$
\left.\begin{array}{l}
\varphi_{1}=A_{1} \cos ^{a_{1}} x \cos ^{b_{1}} y \cos ^{c_{1}} z, \quad \varphi_{2}=A_{2} \cos ^{a_{2}} x \cos ^{b_{2}} y \cos ^{c_{2}} z, \\
\varphi_{3}=A_{3} \cos x \cos y \cos z,
\end{array}\right\}
$$

where

$$
\left.\begin{array}{l}
a_{1}=d_{0}, \quad a_{2}=1-d_{0}, \quad b_{1}=d_{0}-\frac{2 \cos \theta}{A_{1} A_{2}}, \quad b_{2}=1-d_{0}+\frac{2 \cos \theta}{A_{1} A_{2}}, \\
c_{1}=d_{0}+\frac{\sqrt{3} \sin \theta-\cos \theta}{A_{1} A_{2}}, \quad c_{2}=1-d_{0}-\frac{\sqrt{3} \sin \theta-\cos \theta}{A_{1} A_{2}}, \\
A_{3}=A_{1} A_{2}\left(1-d_{0}\right)-\frac{\sqrt{3}}{3} \sin \theta+\cos \theta,
\end{array}\right\}
$$


Vortex-surface fields

Global existence when $H=0$

Global existence when $H \neq 0$

Satisfying $\omega \cdot \nabla \phi=0$ when $H=0$

Requirement on the global smoothness

Possible
Possible
Yes
Yes

Possible

Yes

Yes
Clebsch potentials

Possible

No

Yes

No

TABLE 1. Comparisons between vortex-surface fields and classical Clebsch potentials.
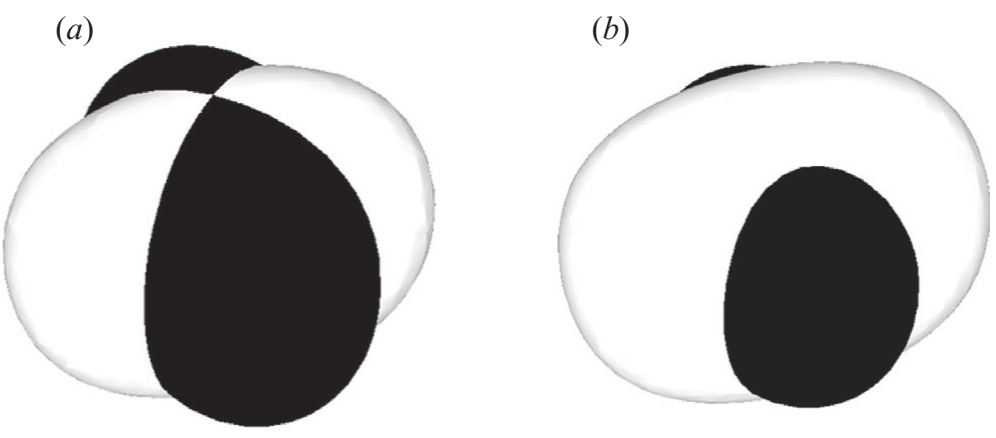

FIGURE 5. Examples of the vortex lines in figure 1(a) expressed by intersections of iso-surfaces of two independent vortex-surface fields, $\phi^{(1)}=\cos x \cos ^{3} y \cos ^{2} z$ (dark surface) and $\phi^{(2)}=\cos ^{3} x \cos y \cos ^{2} z$ (light surface), for the TG initial field (2.9) with $\theta=0$. (a) $\phi^{(1)}=0.5$ and $\phi^{(2)}=0.5$ and $(b) \phi^{(1)}=0.5$ and $\phi^{(2)}=0.4$.

with arbitrary constants $d_{0}, A_{1}$ and $A_{2}$. We can verify that (2.30) must have singularities or imaginary values in some spatial domains. Taking $\theta=0, d_{0}=1$ and $A_{1}=A_{2}=\sqrt{2}$ for example, (2.30) becomes $\varphi_{1}=\sqrt{2} \cos x \sqrt{\cos z}$ and $\varphi_{2}=\sqrt{2} \cos y \sqrt{\cos z}$. This particular solution was given in Nore, Abid \& Brachet (1997), and the sign function was then introduced to remove imaginary values to obtain TG Clebsch potentials with weak singularities.

It is difficult to determine Clebsch potentials from approximate numerical solutions for vortex surface fields such as those obtained presently for the KP flow in $\S 2.3$. Explicit KP Clebsch potentials with singularities or imaginary values are known (Ohkitani 2008) but are generally not useful for the construction of smooth vortexsurface fields for investigating the evolutionary geometry of the vorticity in physical space.

Finally, a comparison of some properties of vortex-surface fields and Clebsch potentials is given in table 1. Both vortex-surface fields and Clebsch potentials exist perhaps only in some subsets of general flows, in particular, flows with the simple topology and geometry.

\section{Evolution of Lagrangian and vortex-surface fields}

\subsection{Evolution equations}

We define a Lagrangian field $\phi(x, t)$ as one for which iso-surfaces $\phi=C$, for each constant $C, \phi_{\min } \leqslant C \leqslant \phi_{\max }$, are always composed of the same particles. Hence, $\phi$ 
satisfies

$$
\frac{\mathrm{D} \phi}{\mathrm{D} t}=\frac{\partial \phi}{\partial t}+\boldsymbol{u} \cdot \nabla \phi=0
$$

Iso-surfaces of $\phi$ are then referred to as material or Lagrangian surfaces. In this section, for a given velocity/vorticity field, we use $\phi_{v}(\boldsymbol{x}, t)$ to denote a vortex-surface field, which we distinguish from the Lagrangian field $\phi(x, t)$. In the sequel, these two fields will generally be taken to be identical at an initial time $t=0$.

Now, in an incompressible flow, consider $\boldsymbol{\omega}(\boldsymbol{x}, t)$ and $\phi_{v}(\boldsymbol{x}, t)$ that, respectively, satisfy

$$
\begin{aligned}
\frac{\mathrm{D} \boldsymbol{\omega}}{\mathrm{D} t} & =\boldsymbol{\omega} \cdot \nabla \boldsymbol{u}+v \nabla^{2} \boldsymbol{\omega}, \\
\frac{\mathrm{D} \phi_{v}}{\mathrm{D} t} & =L_{1}+v \mathscr{L},
\end{aligned}
$$

where $v$ is the kinematic viscosity. In order to define an evolution of a vortex-surface field, we seek $\phi_{v}(\boldsymbol{x}, t)$ with $L_{1}$ and $\mathscr{L}$ such that

$$
\frac{\mathrm{D}}{\mathrm{D} t}\left(\omega \cdot \nabla \phi_{v}\right)=0
$$

with an initial condition that satisfies

$$
\boldsymbol{\omega} \cdot \nabla \phi_{v}(\boldsymbol{x}, t=0) \equiv 0,
$$

for all $\boldsymbol{x}$. It follows that if (3.5) is satisfied at $t=0$, then it is preserved for $t>0$. Hence, $\phi_{v}(\boldsymbol{x}, t)$ evolves as a vortex-surface field. It is noted that (3.4) is equivalent to Ertel's theorem (Ertel 1942) when $\phi_{v}=\phi$, and it also satisfies (3.1).

From (3.2), it can be shown that

$$
\frac{\mathrm{D}}{\mathrm{D} t}\left(\boldsymbol{\omega} \cdot \nabla \phi_{v}\right)=\nu \nabla \phi_{v} \cdot \nabla^{2} \boldsymbol{\omega}+\boldsymbol{\omega} \cdot \nabla\left(\frac{\mathrm{D} \phi_{v}}{\mathrm{D} t}\right),
$$

and using (3.3)

$$
\frac{\mathrm{D}}{\mathrm{D} t}\left(\omega \cdot \nabla \phi_{v}\right)=v \nabla \phi_{v} \cdot \nabla^{2} \omega+\omega \cdot \nabla\left(L_{1}+v \mathscr{L}\right) .
$$

Comparing (3.7) with (3.4), it follows that for $\phi_{v}(\boldsymbol{x}, t)$ to describe the evolution of a vortex-surface field, we require that

$$
v\left(\nabla \phi_{v} \cdot \nabla^{2} \omega+\omega \cdot \nabla \mathscr{L}\right)+\omega \cdot \nabla L_{1}=0 .
$$

\subsection{Inviscid incompressible flow}

First consider the inviscid case $v=0$. Equation (3.8) becomes

$$
\omega \cdot \nabla L_{1}=0,
$$

with solution $L_{1}(\boldsymbol{x})=F\left(\phi_{v}(\boldsymbol{x}, t)\right)$, where $F$ is any smooth function. Hence, $\phi_{v}(\boldsymbol{x}, t)$ must evolve as

$$
\frac{\mathrm{D} \phi_{v}}{\mathrm{D} t}=F\left(\phi_{v}(\boldsymbol{x}, t)\right) .
$$

Comparison of (3.10) with (3.1) shows that the choice $F=0$ makes $\phi_{v}$ a Lagrangian field for $t>0$. This is the Helmholtz vorticity theorem. In our terminology, this states that a Lagrangian surface (Lagrangian field) that is a vortex surface (vortex-surface field) at some initial time remains so for all time. Hence, to track the evolution of a vortex-surface field in an inviscid flow, it is sufficient to determine $\phi_{v}(\boldsymbol{x}, t=0)$ that 
satisfies (3.5), choose $\phi(\boldsymbol{x}, t=0)=\phi_{v}(\boldsymbol{x}, t=0)$ and solve (3.1) for $t>0$ along with the velocity/vorticity dynamics.

Any non-zero $F\left(\phi_{v}\right)$, however, also provides an evolution for a vortex-surface field with the initial condition $\phi_{v}(\boldsymbol{x}, t=0)$ that satisfies (3.5). Iso-surfaces of $\phi_{v}(\boldsymbol{x}, t)$ then remain vortex surfaces for $t>0$, but $\phi_{v}=C$ for some $C$ is then not a Lagrangian surface and so $\phi_{v}$ is not a Lagrangian field. This demonstrates non-uniqueness of the evolution of a vortex-surface field from an initial condition that satisfies (3.5). Thus, an initial vortex-surface field does not have a unique evolution for $t>0$, but there exists a unique evolution for which $\phi_{v}$ is also a Lagrangian field, namely $F=0$. In other words, in an inviscid incompressible flow, a Lagrangian field that was initially a vortex-surface field remains so for all time. But one can define the evolution of vortex-surface fields that are not Lagrangian fields. We remark that this non-uniqueness appears to be distinct from that associated with the definition of $\phi_{v}$ at $t=0$. The latter is a relabelling.

We can, however, show that solutions to (3.10) for a reasonable but arbitrary choice of $F$ are equivalent to a relabelling of the Lagrangian field from the same initial condition. First consider $\phi(\boldsymbol{x}, t)$ satisfying (3.1) with $\phi(\boldsymbol{x}, t=0)=\phi_{0}(\boldsymbol{x})$, given $\phi_{0}$, and second, $\phi_{v}(\boldsymbol{x}, t)$ satisfying (3.10) given $F\left(\phi_{v}\right)$. Choose $\phi_{v}(\boldsymbol{x}, t=0)=\phi_{0}$. Next consider the change of variables $\phi_{v} \leftrightarrow \phi$ given by

$$
G\left(\phi_{v}\right)=t+G(\phi), \quad G(\eta) \equiv \int \frac{\mathrm{d} \eta}{F(\eta)},
$$

where $F(\phi)$ satisfies the (strong) condition that (3.11) is regular and $1-1$ for all $t$. Then, it is straightforward to show that under (3.11), (3.10) is mapped to (3.1). Further, from (3.11), at $t=0, \phi_{v}(\boldsymbol{x}, t=0)=\phi(\boldsymbol{x}, t=0)=\phi_{0}(\boldsymbol{x})$. It follows that, given a solution at time $t$ of (3.1) with $\phi(x, t=0)=\phi_{0}(\boldsymbol{x})$, the solution of (3.10) at time $t$ with the same initial condition is

$$
\phi_{v}(\boldsymbol{x}, t)=G^{-1}(t+G(\phi(\boldsymbol{x}, t)),
$$

where $G^{-1}$ denotes the inverse function. In particular, iso-surfaces of $\phi_{v}$ and $\phi$ are mapped into each other by (3.12). Non-uniqueness then exists only up to a relabelling: at $t>0$, the fields $\phi_{v}$ and $\phi$ are geometrically isomorphic in that the geometry of iso-surfaces for respective constants satisfying (3.11) is identical. In this sense, $F$ has been scaled out of the evolution. As an example, the choice $F=\phi_{v}$ gives $\phi_{v}(\boldsymbol{x}, t)=\exp (t) \phi(\boldsymbol{x}, t)$ and is regular for all $t$. We remark that choices of $F$ for which (3.11) is singular for some $t>0$ implies that the evolution of $\phi_{v}$ can develop a singularity in a finite time. An example is $F=\phi_{v}^{2}$. This has no physical significance.

\subsection{Viscous incompressible flow}

In the viscous case, $v>0$, we can, without loss of generality, either put $L_{1} \equiv 0$ or absorb $L_{1}$ into $\mathscr{L}$ in (3.8) so that, for $\phi_{v}(\boldsymbol{x}, t)$ to be a vortex-surface field for $t>0$, we must solve, along with the velocity-vorticity equations,

$$
\frac{\mathrm{D} \phi_{v}}{\mathrm{D} t}=v \mathscr{L}
$$

where $\mathscr{L}\left(\phi_{v}, \omega\right)$ is determined by

$$
\nabla \phi_{v} \cdot \nabla^{2} \omega+\omega \cdot \nabla \mathscr{L}=0 .
$$

While there is clearly no solution for $\phi_{v}$ which is a Lagrangian field, given $\omega$ and $\phi_{v}$ satisfying (3.5) at some $t$, say $t=0$, it is of interest to enquire if there exist solutions 
to (3.14) that define the evolution of $\phi_{v}(\boldsymbol{x}, t)$ as a vortex-surface field for $t>0$. In particular, can a unique solution be defined that is, in some sense, the continuation in $v>0$ of the inviscid case? Equation (3.14) is an inhomogeneous, non-constant coefficient equation for $\mathscr{L}$. If solutions exist, it is easy to show that they are not unique: for if $\mathscr{L}_{p}$ is a particular solution, then since by (3.5) the homogeneous form of (3.14) is satisfied by an arbitrary function $H\left(\phi_{v}\right), \mathscr{L}_{p}+H\left(\phi_{v}\right)$ is also a solution. Unlike the inviscid case, it appears that this cannot be reduced to a geometrical isomorphism.

Furthermore, from (3.2), (3.13) and (3.14), we can obtain transport equations for $|\omega|$ and $\left|\nabla \phi_{v}\right|$

$$
\begin{aligned}
\frac{\mathrm{D}|\boldsymbol{\omega}|}{\mathrm{D} t} & =\left(\boldsymbol{n}_{\omega} \cdot \boldsymbol{S} \cdot \boldsymbol{n}_{\omega}+v \boldsymbol{R}_{\omega} \boldsymbol{n}_{\omega} \cdot \boldsymbol{n}_{\Delta \omega}\right)|\boldsymbol{\omega}|, \\
\frac{\mathrm{D}\left|\nabla \phi_{v}\right|}{\mathrm{D} t} & =\left(-\boldsymbol{n}_{v} \cdot \boldsymbol{S} \cdot \boldsymbol{n}_{v}-v \boldsymbol{R}_{\omega} \boldsymbol{n}_{\omega} \cdot \boldsymbol{n}_{\Delta \omega}\right)\left|\nabla \phi_{v}\right|,
\end{aligned}
$$

where the rate-of-strain tensor $\boldsymbol{S}$ has entries $S_{i j}=\left(\partial u_{i} / \partial x_{j}+\partial u_{j} / \partial x_{i}\right) / 2$, and unit vectors and a dimensionless ratio, respectively, are

$$
\boldsymbol{n}_{\omega}=\frac{\boldsymbol{\omega}}{|\boldsymbol{\omega}|}, \quad \boldsymbol{n}_{\Delta \omega}=\frac{\nabla^{2} \boldsymbol{\omega}}{\left|\nabla^{2} \boldsymbol{\omega}\right|}, \quad \boldsymbol{n}_{v}=\frac{\nabla \phi_{v}}{\left|\nabla \phi_{v}\right|}, \quad R_{\omega}=\frac{\left|\nabla^{2} \boldsymbol{\omega}\right|}{|\boldsymbol{\omega}|} .
$$

The evolution of the spatial averaged $|\omega|$ and $\left|\nabla \phi_{v}\right|$ can then be expressed as

$$
\begin{aligned}
\langle|\omega(t)|\rangle & =\left\langle\left|\omega_{0}\right|\right\rangle \exp \left(\int_{t 0}^{t}\left\langle\boldsymbol{n}_{\omega} \cdot \boldsymbol{s} \cdot \boldsymbol{n}_{\omega}\right\rangle+\left\langle v R_{\omega} \boldsymbol{n}_{\omega} \cdot \boldsymbol{n}_{\Delta \omega}\right\rangle \mathrm{d} t^{\prime}\right), \\
\left\langle\left|\nabla \phi_{v}(t)\right|\right\rangle & =\left\langle\left|\nabla \phi_{v 0}\right|\right\rangle \exp \left(\int_{t 0}^{t}-\left\langle\boldsymbol{n}_{v} \cdot \boldsymbol{S} \cdot \boldsymbol{n}_{v}\right\rangle-\left\langle v R_{\omega} \boldsymbol{n}_{\omega} \cdot \boldsymbol{n}_{\Delta \omega}\right\rangle \mathrm{d} t^{\prime}\right) .
\end{aligned}
$$

Here, in general viscous flows, statistically the enstrophy production rate $\left\langle\boldsymbol{n}_{\omega} \cdot \boldsymbol{S} \cdot \boldsymbol{n}_{\omega}\right\rangle$ in (3.18) is positive owing to predominant vortex stretching. It follows that the viscous term $\left\langle v R_{\omega} \boldsymbol{n}_{\omega} \cdot \boldsymbol{n}_{\Delta \omega}\right\rangle$ should be negative to keep $\langle|\boldsymbol{\omega}|\rangle$ finite. But this implies that the same viscous term would increase $\left\langle\left|\nabla \phi_{v}\right|\right\rangle$ in (3.19). Without the viscous mechanism, it appears to be difficult to give an estimation on the upper bound of $\left\langle\left|\nabla \phi_{v}\right|\right\rangle$, which may cause numerical issues in the computation of evolving vortex-surface fields in a viscous flow.

Thus, for a viscous flow with TG and KP initial conditions, we will presently follow Lagrangian fields $\phi$ whose evolution is defined by (3.1) and which are vortexsurface fields at $t=0$, as determined in $\S 2$. We will show that, for small viscosity or large Reynolds number, such a Lagrangian field can remain a good approximation to a vortex-surface field before significant topological changes, for example vortex reconnection. The problem of the evolution of vortex-surface fields in a viscous flow at low Reynolds numbers is discussed further in Appendix C.

\section{Numerical methods}

\subsection{Eulerian velocity field in $T G$ and KP flows}

A vortex-surface field as defined presently can be considered as a Lagrangian field only in an inviscid flow. However, considering the requirement of huge computational resources and open issues concerning unresolved computations in the simulation of the Euler equation (see Hou \& Li 2008), in $\S 5$, we will investigate the approximation of vortex surfaces in a slightly viscous flow. The corresponding Navier-Stokes equations 


\begin{tabular}{lrc} 
Run & \multicolumn{1}{c}{ Re } & $N^{3}$ \\
TG1 & 200 & $256^{3}$ \\
TG2 & 1600 & $512^{3}$ \\
KP1 & 500 & $512^{3}$ \\
KP2 & 1000 & $512^{3}$
\end{tabular}

TABLE 2. Parameters of the study.
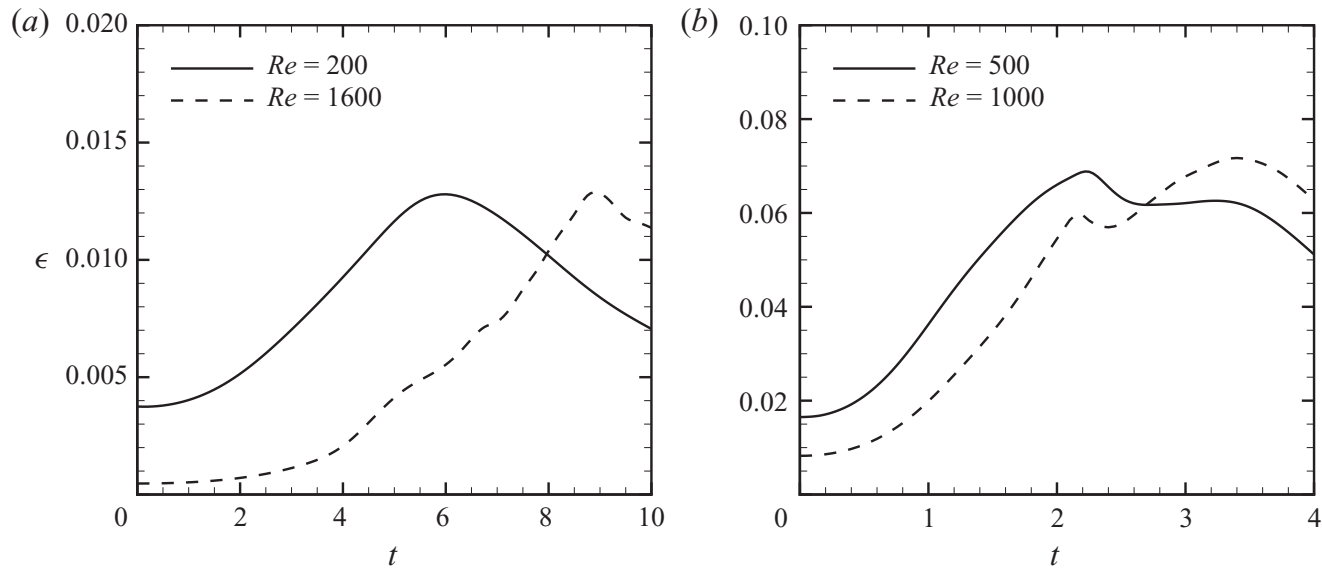

FIGURE 6. Dissipation rate. (a) TG flow and $(b)$ KP flow.

for incompressible TG and KP flows in a periodic box of side $L=2 \pi$ are written in the general form

$$
\left.\begin{array}{l}
\frac{\partial \boldsymbol{u}}{\partial t}=\boldsymbol{u} \times \boldsymbol{\omega}-\nabla\left(\frac{p}{\rho}+\frac{1}{2}|\boldsymbol{u}|^{2}\right)+v \nabla^{2} \boldsymbol{u}, \\
\nabla \cdot \boldsymbol{u}=0,
\end{array}\right\}
$$

where $p$ denotes the pressure and $\rho$ the density.

The numerical simulations were performed using a standard pseudo-spectral method. The flow domain was discretized uniformly into $N^{3}$ grid points. Aliasing errors were removed using the two-thirds truncation method. The Fourier coefficients of the flow velocity were advanced in time using a second-order Adams-Bashforth method for the nonlinear term and an exact integration for the linear viscous term. The time step was chosen to ensure that the Courant-Friedrichs-Lewy number was 0.25 or less for numerical stability and accuracy. An important feature of both flows is that the dissipation rate $\epsilon=v\langle\nabla \boldsymbol{u}: \nabla \boldsymbol{u}\rangle$ is increased at early time and approaches a peak after a finite time, as shown in figure 6. This reproduced the results in Brachet et al. (1983) and Boratav \& Pelz (1994). More details about the computation of TG and KP flows can be found in these two references. Parameters of runs carried out in this study are listed in table 2 , where $R e=1 / v$, considering that the length and velocity scales in (2.9) and (2.14) are $O(1)$.

\subsection{Lagrangian field}

To solve the governing equation (3.1) of the Lagrangian field, the backwardparticle-tracking method, which is stable, topology-preserving and without numerical dissipation, was used. Details of this method are demonstrated in Yang et al. (2010). 
Briefly, (3.1) is converted to a set of ODEs in a Lagrangian sense to compute trajectories of fluid particles as

$$
\frac{\partial \boldsymbol{X}\left(\boldsymbol{x}_{0}, t_{0} \mid t\right)}{\partial t}=\boldsymbol{V}\left(\boldsymbol{x}_{0}, t_{0} \mid t\right)=\boldsymbol{u}\left(\boldsymbol{X}\left(\boldsymbol{x}_{0}, t_{0} \mid t\right), t\right),
$$

where $\boldsymbol{X}\left(\boldsymbol{x}_{0}, t_{0} \mid t\right)$ is the location at time $t$ of the fluid particle which was located at $\boldsymbol{x}_{0}$ at the initial time $t_{0}$ and $\boldsymbol{V}\left(\boldsymbol{x}_{0}, t_{0} \mid t\right)$ the velocity at time $t$ of the fluid particle, which is equivalent to tracing characteristics of the hyperbolic equation (3.1). First, the Navier-Stokes equations (4.1) are solved numerically on the $N^{3}$ grid in some time interval $t_{0}, t>t_{0}$, and the full Eulerian velocity field in the impermeable box is saved on disk at every time step over this simulation period. The time increment is selected to capture the finest resolved scales in the velocity field. At time $t$ at the end of the solution period, particles are placed at the grid points of $N_{p}^{3}$ in the periodicity box. Here, $N_{p}$ should be greater than the grid number $N$ of the velocity field, i.e. the resolution of the Lagrangian field should be higher than the velocity field to capture some nearly singular Lagrangian structures in the evolution (Yang et al. 2010). Then, particles are released and their trajectories calculated by solving (4.2) backwards in time. A three-dimensional fourth-order Lagrangian interpolation scheme was used to calculate fluid velocity at the location of a particle. The trajectory of a particle was then obtained by the explicit second-order Adams-Bashforth scheme. For each particle, the backward tracking is performed from $t$ to the initial time $t_{0}$ with the reversed Eulerian velocity fields saved previously. When particles run out of the impermeable box, the reflectional symmetries in $\boldsymbol{u}(\boldsymbol{x}, t)$ need to be considered (see Brachet et al. 1983). After the backward tracking, initial locations of particles $\boldsymbol{x}_{0}$ can be obtained. From a given initial condition consisting of a smooth Lagrangian field $\phi\left(\boldsymbol{x}_{0}, t_{0}\right)$, we can then obtain the Lagrangian field at time $t$ on the Cartesian grid by a simple mapping with Lagrangian coordinates:

$$
\phi(x, t)=\phi\left(X\left(x_{0}, t_{0} \mid t\right), t\right) \longleftrightarrow \phi\left(x_{0}, t_{0}\right)
$$

Next, initial conditions $\phi_{T G}$ and $\phi_{K P}$ developed in $\$ \S 2.2$ and 2.3 are, respectively, applied to obtain the corresponding Lagrangian field in TG and KP flows at the particular time by (4.3). Additional interpolations from $\phi_{K P}$ are required in the computation of (4.3) for the KP flow. Taking an advantage of symmetries in both flows, we can simulate $\phi$ only in the fundamental box with resolution $\left(N_{p} / 4+1\right)^{3}$, where $N_{p}=4096$ in the present study. Then, $\phi$ in the impermeable box can be reconstructed by following steps with rotational symmetries:

$$
\begin{aligned}
& \phi(x, y, z)=-\phi(y, \pi-x, z), \quad \pi / 2 \leqslant x \leqslant \pi, \quad 0 \leqslant y \leqslant \pi / 2, \quad 0 \leqslant z \leqslant \pi / 2, \\
& \phi(x, y, z)=-\phi(\pi-y, x, z), \quad 0 \leqslant x \leqslant \pi / 2, \quad \pi / 2 \leqslant y \leqslant \pi, \quad 0 \leqslant z \leqslant \pi / 2 \\
& \phi(x, y, z)=\phi(\pi-x, \pi-y, z), \quad \pi / 2 \leqslant x \leqslant \pi, \quad \pi / 2 \leqslant y \leqslant \pi, \quad 0 \leqslant z \leqslant \pi / 2, \\
& \phi(x, y, z)=\phi(x, \pi-y, \pi-z), \quad 0 \leqslant x \leqslant \pi, \quad 0 \leqslant y \leqslant \pi, \quad \pi / 2 \leqslant z \leqslant \pi .
\end{aligned}
$$

Finally, $\phi$ in the periodicity box can be obtained from $\phi$ in the impermeable box by reflectional symmetries with the planes $x, y, z=\pi$. In addition, $\phi$ can be simulated only in the fundamental tetrahedron for the KP flow to make a further reduction on the computation size. 


\section{Numerical simulation and results}

5.1. Error estimation in the evolution of the vortex-surface field

If the flow is inviscid, an iso-surface of the Lagrangian field $\phi(x, t)$ is a vortex surface. When the viscosity is non-zero, even very small, a Lagrangian surface that was a vortex surface at the initial time will, in general, no longer remain a vortex surface. Iso-surfaces of $\phi$, however, which started as vortex surfaces, could still be a good approximation to a vortex surface within a finite time for a high- $R e$ flow. We presently investigate this approximation.

A measure of the deviation of a Lagrangian surface $\phi=$ constant from a vortex surface $\phi_{v}=$ constant is the cosine of the angle $\chi$ between $\omega$ and $\nabla \phi$ :

$$
\lambda_{\omega} \equiv \cos \chi=\frac{\omega \cdot \nabla \phi}{|\omega||\nabla \phi|} \text {. }
$$

Using (3.1) and (3.2) and replacing $\phi_{v}$ by $\phi$ in (3.6) provide a corresponding transport equation for $\lambda_{\omega}$,

where

$$
\frac{\mathrm{D} \lambda_{\omega}}{\mathrm{D} t}=\mathscr{A} \lambda_{\omega}+\mathscr{B}
$$

$$
\begin{aligned}
& \mathscr{A}(\boldsymbol{x}, t)=\boldsymbol{n}_{\phi} \cdot \boldsymbol{S} \cdot \boldsymbol{n}_{\phi}-\boldsymbol{n}_{\omega} \cdot \boldsymbol{S} \cdot \boldsymbol{n}_{\omega}-v R_{\omega} \boldsymbol{n}_{\omega} \cdot \boldsymbol{n}_{\Delta \omega}, \\
& \mathscr{B}(\boldsymbol{x}, t)=v \boldsymbol{R}_{\omega} \boldsymbol{n}_{\phi} \cdot \boldsymbol{n}_{\Delta \omega},
\end{aligned}
$$

with notations in (3.17) and $\boldsymbol{n}_{\phi}=\nabla \phi /|\nabla \phi|$. On a fluid particle, the solution of (5.2) has the form of

$$
\lambda_{\omega}(t)=\lambda_{\omega 0} \exp \left(\int \mathscr{A}(t) \mathrm{d} t\right)+\exp \left(\int \mathscr{A}(t) \mathrm{d} t\right) \int \exp \left(-\int \mathscr{A}(t) \mathrm{d} t\right) \mathscr{B}(t) \mathrm{d} t,
$$

with $\lambda_{\omega 0} \equiv \lambda_{\omega}(t=0)$.

For the initial vortex-surface field, $\omega$ is normal to $\nabla \phi$ and $\lambda_{\omega 0}=0$ everywhere, and (5.5) becomes

$$
\lambda_{\omega}(t)=\exp \left(\int \mathscr{A}(t) \mathrm{d} t\right) \int \exp \left(-\int \mathscr{A}(t) \mathrm{d} t\right) \mathscr{B}(t) \mathrm{d} t .
$$

Therefore, $\lambda_{\omega}$ is always zero in an inviscid flow with $\mathscr{B}=0$. In viscous TG and KP flows, although $\mathscr{B}(t=0)=0$ for both $\mathrm{TG}$ and KP initial conditions, the transport equation for $\nabla \phi \cdot \nabla^{2} \omega$,

$$
\frac{\mathrm{D}}{\mathrm{D} t}\left(\nabla \phi \cdot \nabla^{2} \boldsymbol{\omega}\right)=\left(\frac{\partial}{\partial t} \nabla^{2} \boldsymbol{\omega}+\nabla \times\left(\nabla^{2} \boldsymbol{\omega} \times \boldsymbol{u}\right)\right) \cdot \nabla \phi,
$$

implies that $|\mathscr{B}(t)|>0$ and then $\left|\lambda_{\omega}(t)\right|$ should increase with time after $t=0$. The relative error can be defined by the normalized deviation angle between the tangent plane and the vorticity at each of its points on the iso-surface of $\phi$ :

$$
\Delta \chi=(1-2|\chi| / \pi) \times 100 \%, \quad|\chi| \leqslant \pi / 2 .
$$

The time evolution of the volume-averaged quantity $\left\langle\left|\lambda_{\omega}\right|\right\rangle$ in TG and KP flows is plotted in figure 7. Here, $\left\langle\left|\lambda_{\omega}\right|\right\rangle$ for the KP flow is computed only in the region with $\hat{\phi} \geqslant 10^{-3}$ to avoid the effect of errors in the approximate solution $\phi_{K P}$ at small contour levels on the evolution of $\left|\lambda_{\omega}\right|$. Here, the normalized iso-contour level is defined by

$$
\hat{\phi}=\phi /|\phi|_{\max } \text {. }
$$



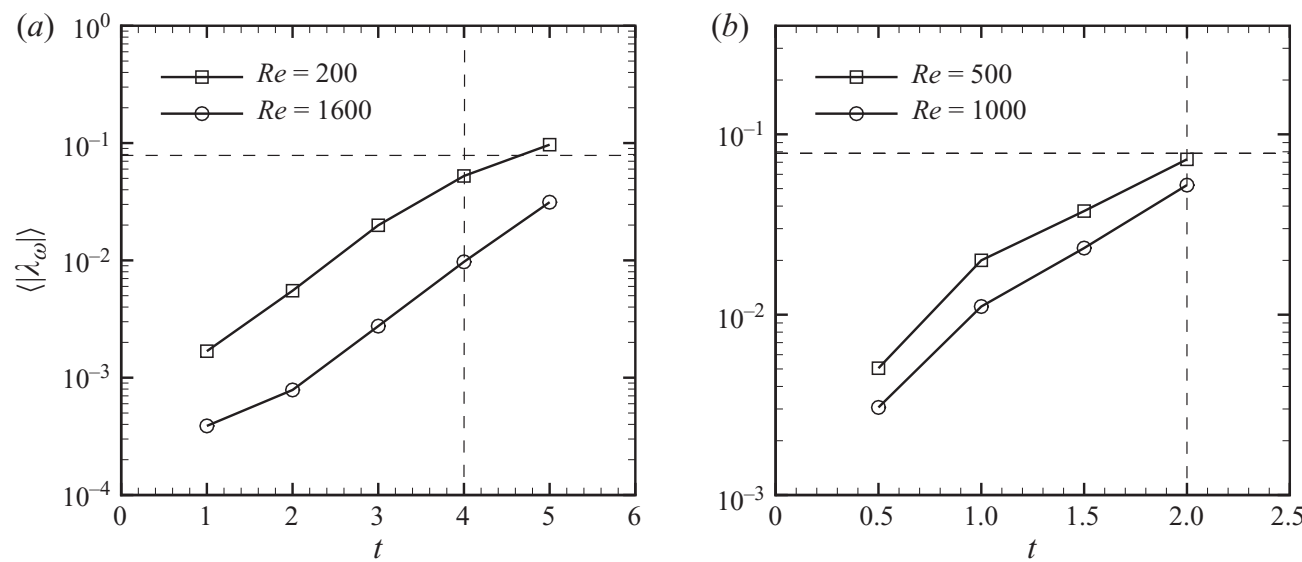

FIGURE 7. Temporal evolution of the volume-averaged $\left|\lambda_{\omega}\right|$. The horizontal dashed line denotes the relative error $\Delta \chi=5 \%$ and the vertical dashed line denotes the approximate potential singularity time $t^{*}$. (a) TG flow and $(b)$ KP flow.

Exponential increase of $\left\langle\left|\lambda_{\omega}\right|\right\rangle$ is observed in both flows at different Reynolds numbers, and $\left\langle\left|\lambda_{\omega}\right|\right\rangle$ in a low-Re flow grows faster than in a high-Re flow due to the effect of viscosity. However, the errors before $t=4$ in the TG flow at $R e=1600$ and $t=2$ in the KP flow at $R e=1000$ are relatively small $(\Delta \chi<5 \%)$. Therefore, we may reasonably assume that vortex surfaces are still well approximated by Lagrangian surfaces at the early stages of both high-Re flows because the vortex dynamics in high-Reynolds-number flows are essentially inviscid (Brachet et al. 1983).

All vortex surfaces at the initial time are simply connected closed surfaces in TG and KP flows. In viscous flows, this topology will be maintained until vortex reconnection. The issue of formation of a finite-time singularity for an inviscid Euler flow remains an important open question (see Majda \& Bertozzi 2001). Without assuming singularity formation for an inviscid flow, we will nonetheless subsequently use the symbol $t^{*}$ to refer to estimates of possible singularity formation time for both TG and KP initial conditions. According to Brachet et al. (1992) and Boratav \& Pelz (1994), $t^{*} \approx 4$ and $t^{*} \approx 2$ for inviscid TG and KP flows, respectively. As pointed out in Kida \& Takaoka (1994), vortex reconnection in the inviscid limit requires the occurrence of a finite-time singularity, which suggests that violent topological changes may occur at about $t^{*}$ in a viscous flow. The topology, however, is invariant for Lagrangian structures. This contradiction appears to lead to noticeable errors ( $\Delta \chi$ is around or larger than $5 \%$ ) near or after $t^{*}$ in figure 7.

The probability density function (p.d.f.) of $\left|\lambda_{\omega}\right|$ in a TG flow at $R e=1600$ (figure $8 a$ ) shows that the distribution of the deviation $\left|\lambda_{\omega}\right|$ at later times, $t=4$ and $t=5$, is intermittent. To investigate the source of the error, $\left|\lambda_{\omega}\right|$ conditioned on the scalar gradient $|\nabla \phi|$ is plotted in figure $8(b)$. When the scalar gradient is high, the deviation is also large accordingly. This observation emphasizes the distinction between Lagrangian surfaces and vortex surfaces when they are in close vicinity within a viscous flow. The topologically invariant Lagrangian surfaces cannot reconnect whereas vortex surfaces can change topology during a reconnection event. The latter then leads to increases in $\left|\lambda_{\omega}\right|$, corresponding to tilting of the vorticity vector off the Lagrangian surface, which are correlated with the corresponding increases of $|\nabla \phi|$ within the Lagrangian field. 

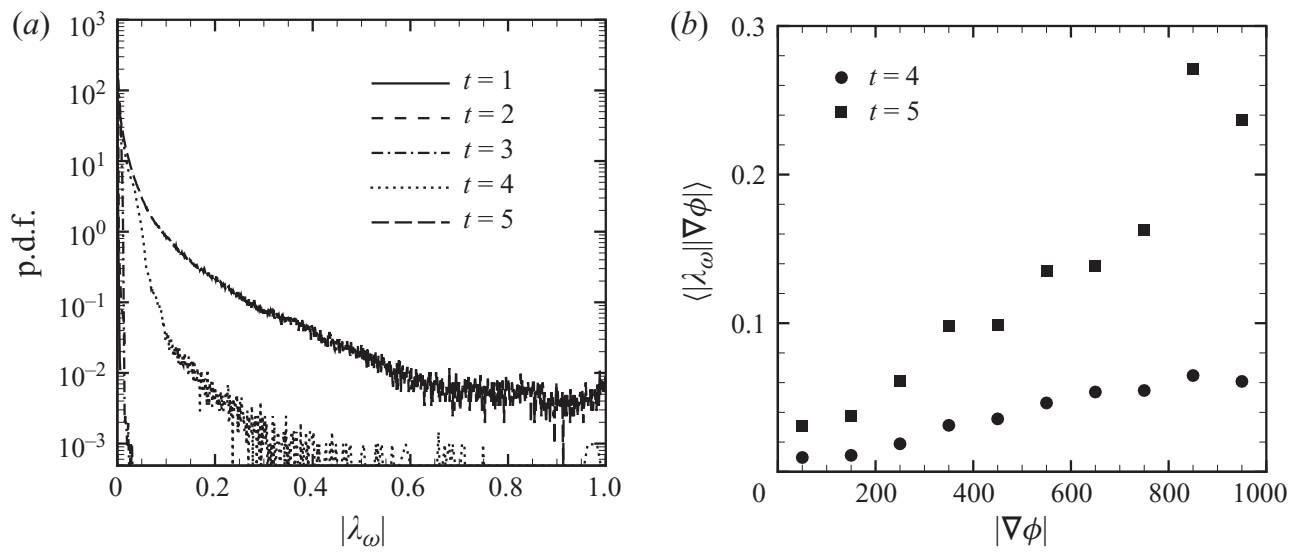

Figure 8. Probability density functions of $\left|\lambda_{\omega}\right|$ at different times $(a)$ and the conditional mean of $\left|\lambda_{\omega}\right|$ on $|\nabla \phi|(b)$ in the TG flow at $R e=1600$.

On the basis of our error estimation, we will next assume that Lagrangian structures that were initially vortex surfaces in high- $R e$ flows, e.g. the TG flow at $R e=1600$ and the KP flow at $R e=1000$ can, before $t^{*}$, be approximated as vortex surfaces with relative error $\Delta \chi<5 \%$. The temporal evolution of cross-sections of a set of vortex surfaces from two views in the TG flow at $R e=1600$ represented by contour lines of $\phi(x, t)$ are shown in figures 9 and 10. The initially blob-like vortex surfaces are progressively distorted into sheet-like surfaces and neighbouring vortex surfaces approach each other. We observe that both the thickness of vortex surfaces and the minimum distance between neighbouring ones decay from $t=0$ to $t=4$. The latter produces high gradients of $\phi$ near $t=t^{*}$ and suggests the onset of vortex reconnection in the viscous flow.

After $t=t^{*}$, in figure 11 we plot two $y-z$ plane-cuts at $x=\pi / 16$ in the fundamental box with resolution $8192^{2}$ for the periodicity box. These show more complicated multi-layered spiral Lagrangian surfaces in the TG flow at the later stages of the numerical simulation. The extremely large $|\nabla \phi|$ generated within these spiral layers is an example of the exponentially small structures produced within the evolving Lagrangian field. The Lagrangian surfaces can then no longer be considered as a good approximation to vortex surfaces.

For long times, both TG and KP flows become decaying turbulence after transitions (Brachet et al. 1983; Boratav \& Pelz 1994). The preferred alignment between the passive scalar gradient and the most compressive strain direction of $\boldsymbol{S}$ in turbulence implies that $\left\langle\boldsymbol{n}_{\phi} \cdot \boldsymbol{S} \cdot \boldsymbol{n}_{\phi}\right\rangle<0$, and the preferred alignment between the vorticity and the intermediate stretching strain direction of $\boldsymbol{S}$ implies that $\left\langle\boldsymbol{n}_{\omega} \cdot \boldsymbol{S} \cdot \boldsymbol{n}_{\omega}\right\rangle>0$ (e.g. Ashurst et al. 1987 and Ruetsch \& Maxey 1992). Thus, in (5.5) and (5.6), statistically $\mathscr{A}(t)<0$ and $\mathscr{B}(t) \sim O\left(v R_{\omega}\right)$ for a long time. Using dimensional analysis, at a moderate Reynolds number, we can obtain an estimate for the inhomogeneous term as

$$
\mathscr{B}(t) \sim \epsilon / E,
$$

where the total energy of flow $E=|\boldsymbol{u}|^{2} / 2$. This implies that, as shown in figures $7(a)$ and 12 , the evolution of $\left|\lambda_{\omega}\right|$ with the initial vortex-surface field $\left|\lambda_{\omega 0}\right|=0$ increases exponentially in the early stage of evolution owing to violation of the Helmholtz theorem in a viscous flow, whereafter the increasing trend is mitigated at later times. 
(a)

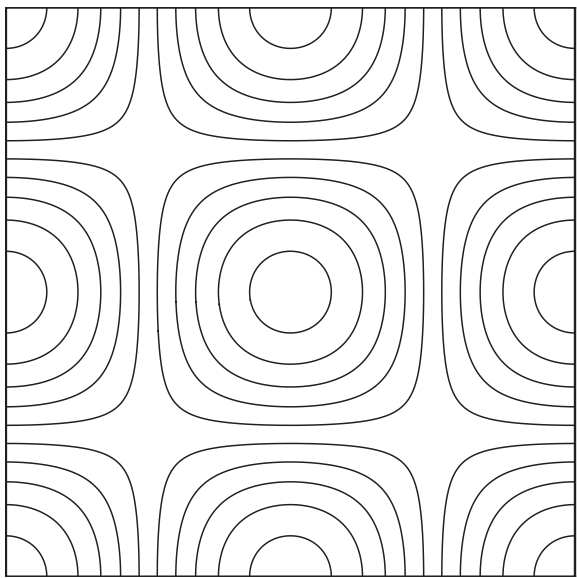

(c)

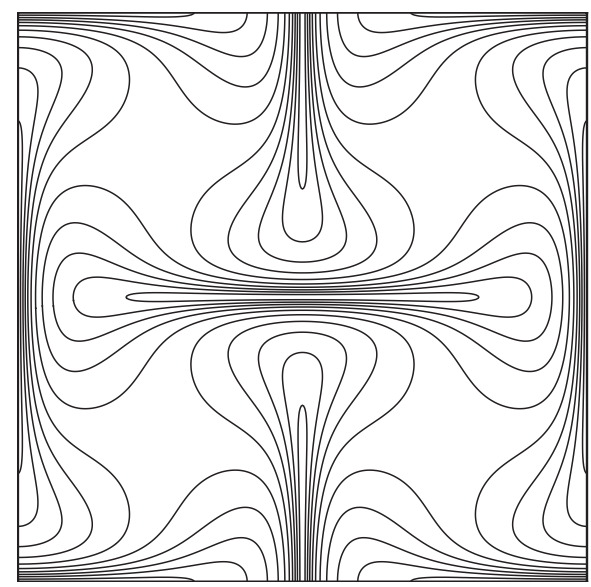

(b)

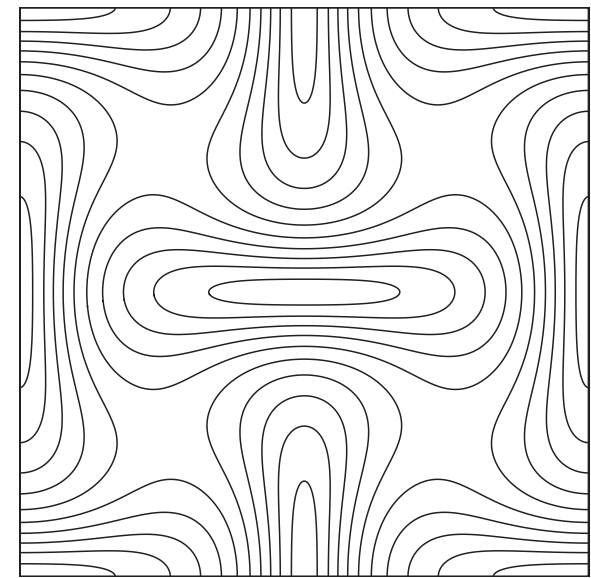

(d)

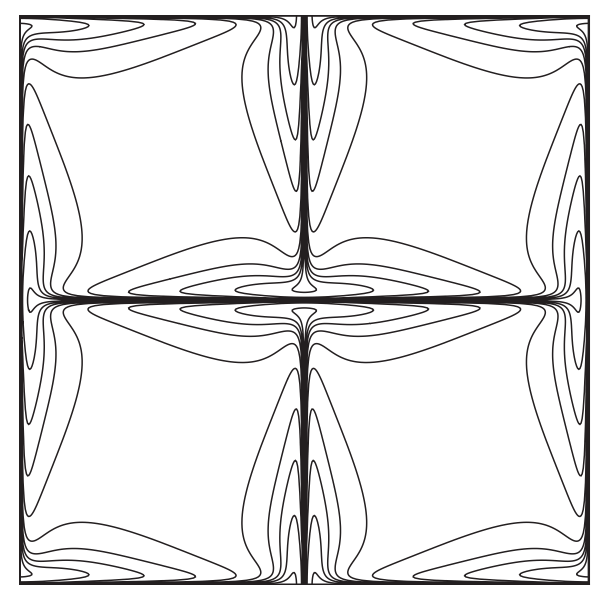

FIGURE 9. Iso-contour lines of $\hat{\phi}=\{ \pm 0.1, \pm 0.3, \pm 0.5, \pm 0.7, \pm 0.9\}$ on the $x-y$ plane-cut at $z=0$ in the periodicity box for the TG flow at $R e=1600$. (a) $t=0,(b) t=1,(c) t=2$ and (d) $t=4$.

Finally, this appears to move towards a stationary stage, which might be controlled by $O(\mathscr{B}(t))$, as shown in figure 12 and implied in (5.6). In contrast, Yang et al. (2010) showed that a Lagrangian field, starting from an arbitrary initial condition with a moderate $\left|\lambda_{\omega 0}\right|$ in stationary isotropic turbulence, appeared to exhibit a tendency to move towards a vortex-surface field of the evolving flow, but this is mitigated by cumulative viscous effects. In the TG flow, from an arbitrary initial Lagrangian field $\phi_{0}=\sin x \sin y \sin z$, we also observed a similar decreasing trend for $\left|\lambda_{\omega}\right|$ in figure 12 . Together, these results suggest that there may exist an intermediate state or 'attractor' for the long-time behaviour of the alignment angle between $\omega$ and $\nabla \phi$ with different initial scalar fields.

\subsection{Geometry of vortex surfaces in evolution}

The geometry in evolution of vortex lines and surfaces is important for quantifying local vorticity growth and the subsequent instability phenomenon and transition in flows with smooth initial conditions. The collective geometry properties of vortex surfaces at a particular time were presently obtained by taking an average over 
(a)

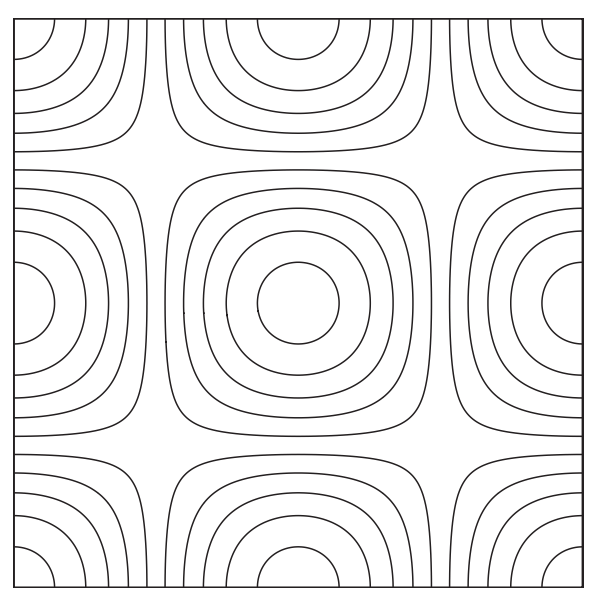

(c)

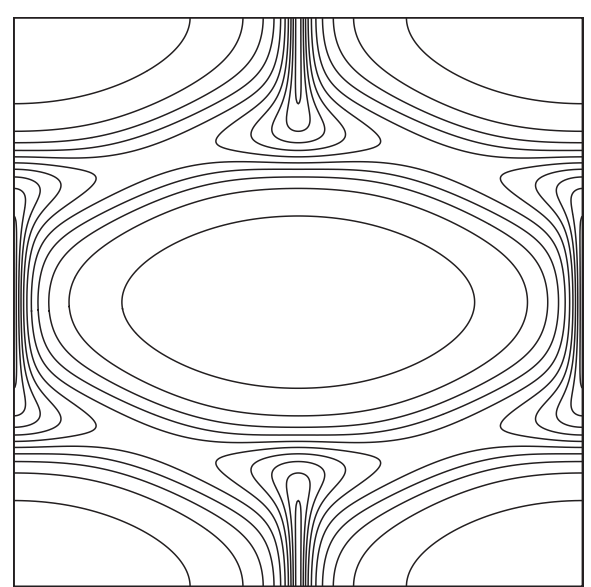

(b)

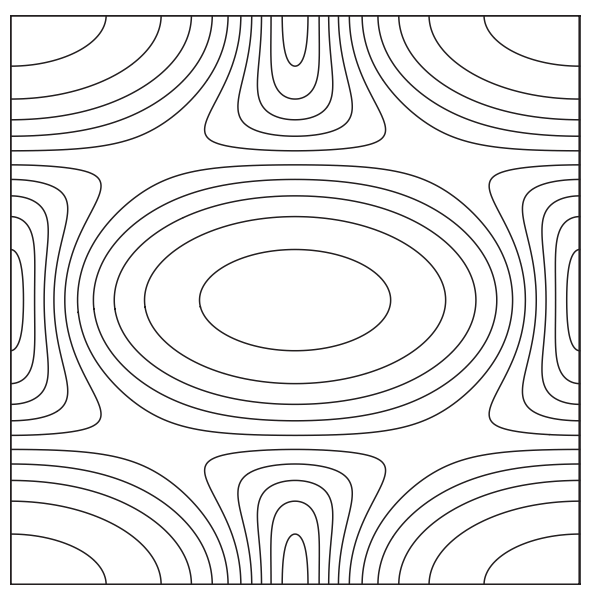

(d)

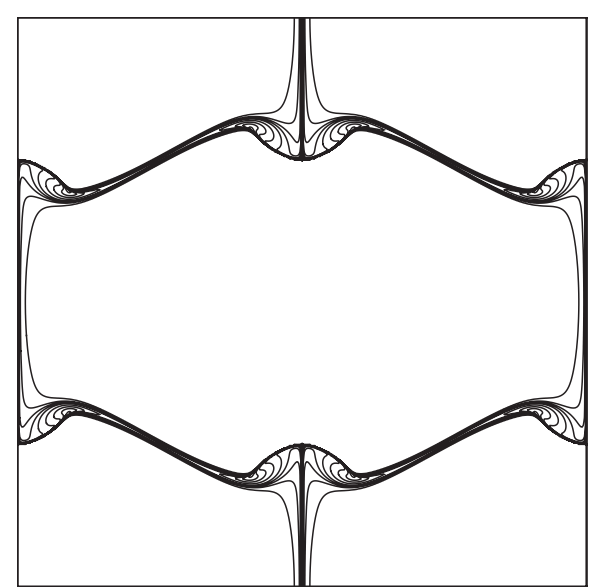

FiguRE 10. Iso-contour lines of $\hat{\phi}=\{ \pm 0.1, \pm 0.3, \pm 0.5, \pm 0.7, \pm 0.9\}$ on the $x-z$ plane-cut at $y=0$ in the periodicity box for the TG flow at $R e=1600$. (a) $t=0,(b) t=1,(c) t=2$ and (d) $t=4$.

(a)

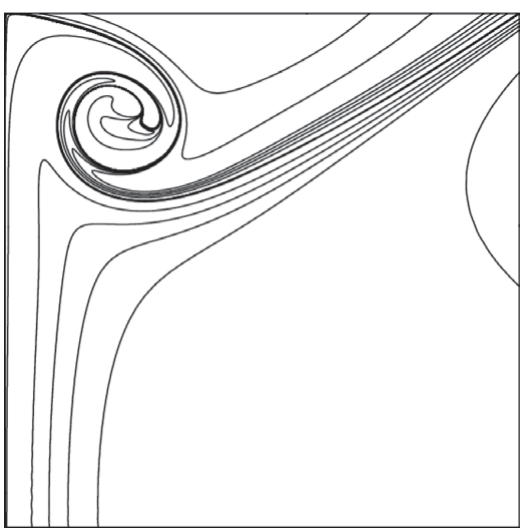

(b)

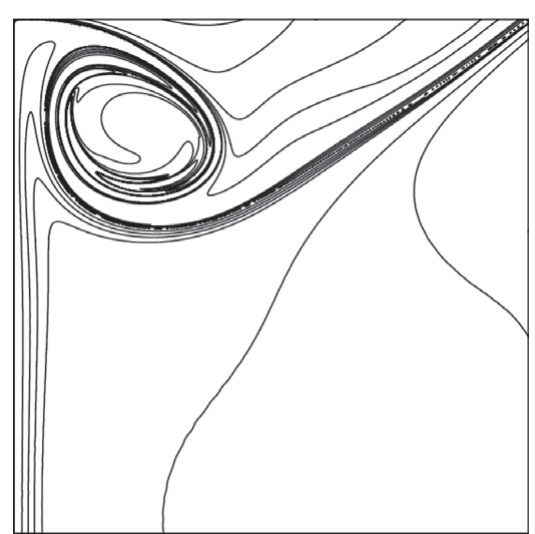

FIGURE 11. Iso-contour lines of $\phi$ on the $y-z$ plane-cut at $x=\pi / 16$ in the fundamental box for the TG flow at $R e=1600$. (a) $t=5$ and $(b) t=6$. 


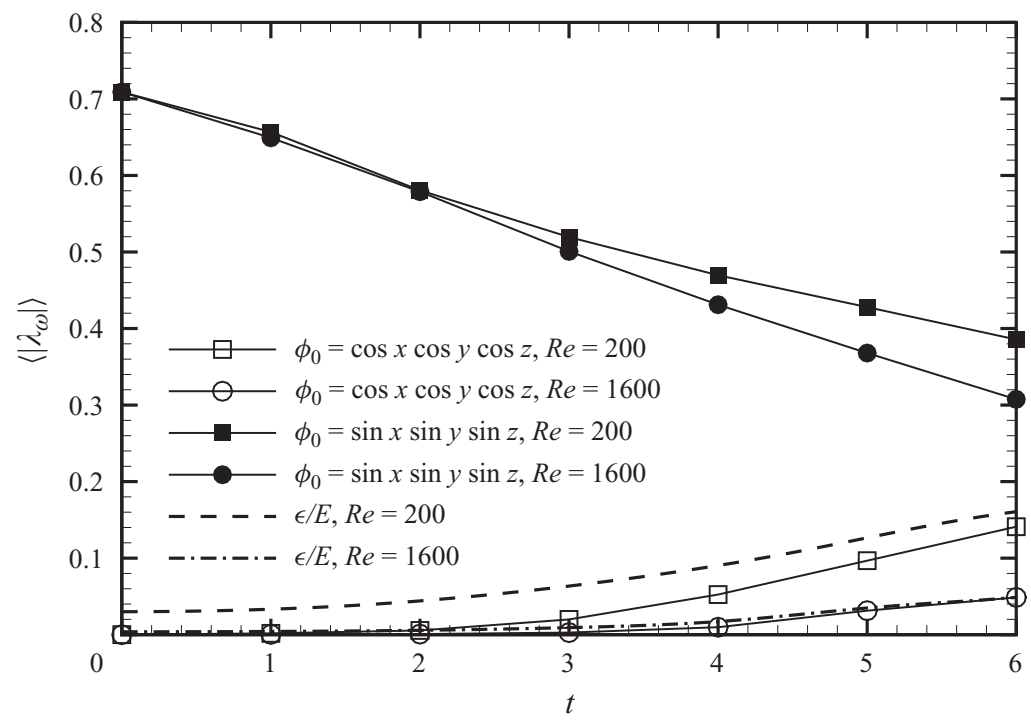

FIGURE 12. Temporal evolution of the volume-averaged $\left|\lambda_{\omega}\right|$ in the TG flow with the initial vortex-surface field $\phi_{0}=\cos x \cos y \cos z$ and an arbitrary initial condition $\phi_{0}=\sin x \sin y \sin z$.
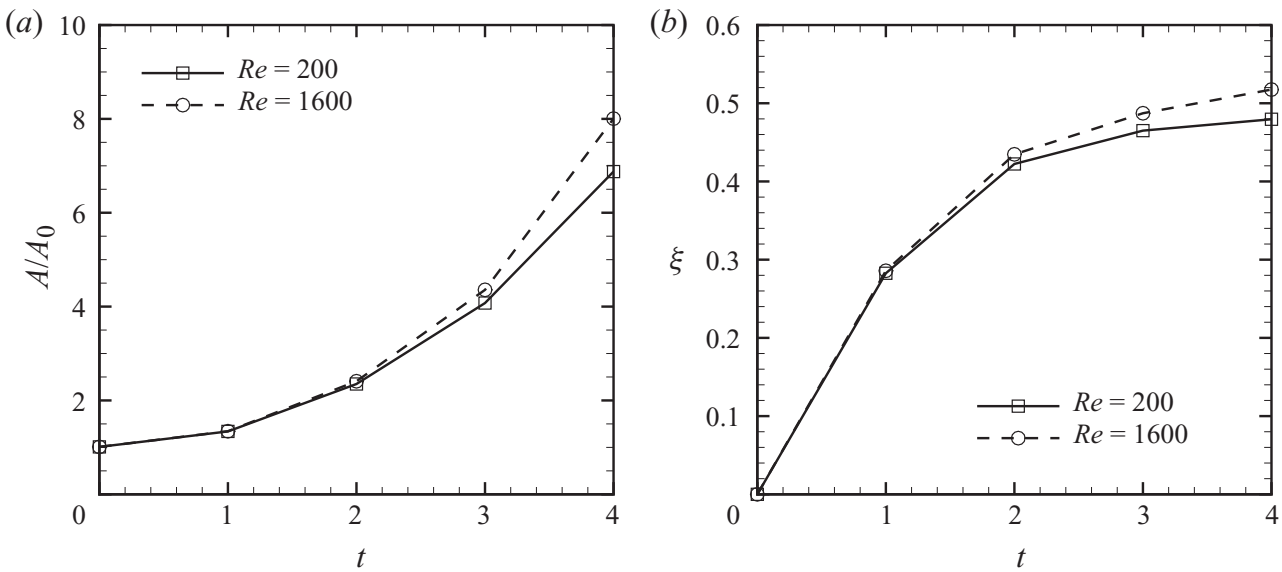

FIGURE 13. Exponential growth of the surface area of vortex surfaces in the TG flow. (a) Surface area and (b) stretching rate.

iso-surfaces of $\phi$ at contour levels $\hat{\phi}=0.1,0.2, \ldots, 0.9$. Since the volume of vortex surfaces is conserved, the surface area $A(t)$ will increase with time in evolution by stretching. The exponential growth of the normalized surface area $A$ by the initial area $A_{0}$, averaged as described above, is shown in figure 13(a), and the corresponding stretching rate,

$$
\xi=\frac{1}{t} \log \left(\frac{A}{A_{0}}\right),
$$

is shown in figure $13(b)$. It is apparent that in the high- $R e$ flow, the stretching rate is higher than the low-Re flow after $t \approx 2$, indicating that the flattening process of vortex surfaces is faster in the higher-Re flow. 


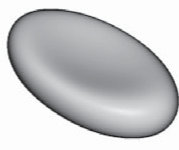

(a)

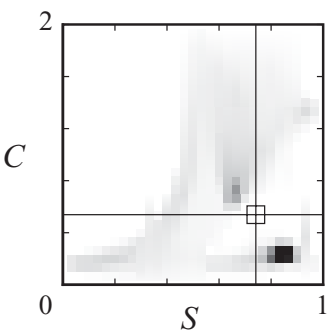

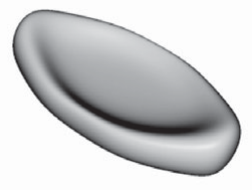

(b)

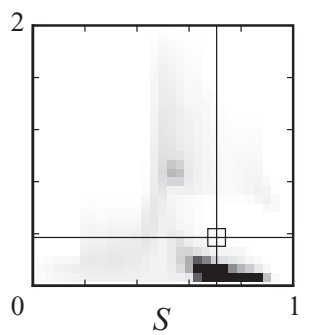

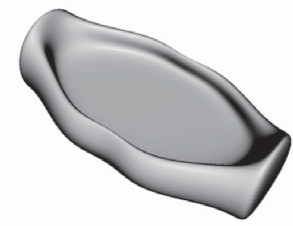

(c)

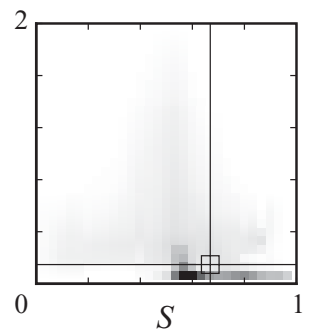

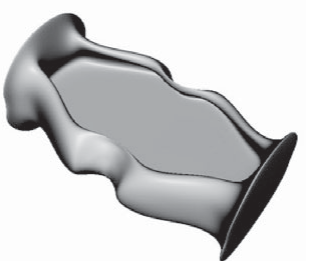

(d)

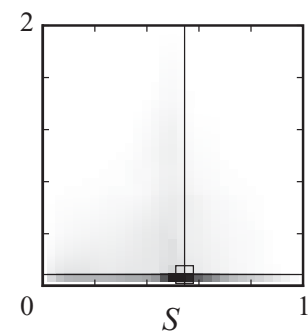

FiguRE 14. Vortex surfaces (iso-surfaces of $\hat{\phi}=0.4$ ) in the TG flow at $R e=1600$ and the corresponding feature centres $(\hat{S}, \hat{C})$ are marked by $\boxplus$ in $S-C$ joint p.d.f.s at the bottom. (a) $t=1,(b) t=2,(c) t=3$ and $(d) t=4$.

In addition to the surface-area growth, measures of curvature on the Lagrangian surface can characterize the local topology. To quantify the non-local geometry (in the surface sense), we utilize two differential geometry properties, the absolute value of the shape index $S$ and dimensionless curvedness $C$ (see Koenderink \& Van Doorn 1992 and Bermejo-Moreno \& Pullin 2008),

$$
S \equiv\left|-\frac{2}{\pi} \arctan \left(\frac{\kappa_{1}+\kappa_{2}}{\kappa_{1}-\kappa_{2}}\right)\right|, \quad C \equiv 3 \frac{V}{A} \sqrt{\frac{\kappa_{1}^{2}+\kappa_{2}^{2}}{2}},
$$

where $\kappa_{1}$ and $\kappa_{2}$ are principal curvatures of a surface, and $V$ is the volume contained within the three-dimensional surface. The local topology can be represented by $S$, where $0 \leqslant S \leqslant 1$. An umbrella-like shape, e.g. the spherical cap or dome, is represented by high $S$; a parabolic shape, e.g. a ridge, is represented by medium $S$; and a hyperbolic shape, e.g. a symmetrical saddle or a saddle ridge, is represented by low $S$ (Koenderink \& Van Doorn 1992). The evolution of three-dimensional vortex surfaces is visualized in figure 14. The corresponding area-based joint p.d.f.s of $S$ and $C$, with colour intensity to express the joint p.d.f. from low to high, are shown below the corresponding visualization of vortex surfaces. The non-local geometry of the surface can be represented by the feature centre $(\hat{S}, \hat{C})$ marked by $\boxplus$ in figure 14 , which are, respectively, dimensionless forms of the weighted first-order moments of the joint p.d.f. of $S$ and $C$. For details, a number of representative shapes and the corresponding $S-C$ joint p.d.f.s are shown in figure 13 in Bermejo-Moreno \& Pullin (2008). The $S-C$ joint p.d.f. at $t=4$ in figure 14 shows that the distribution of $C$ is intermittent. Most surface elements are very flat with low curvatures except for a small number of elements with very high curvatures. From figure $14(d)$, we can see very thin and flat large-scale structures in the middle surrounded by thin edges with very sharp corners. There is a preponderance of surface elements on the vortex surface with hyperbolic or parabolic shapes near $t^{*}$, while most shapes of elements at the 

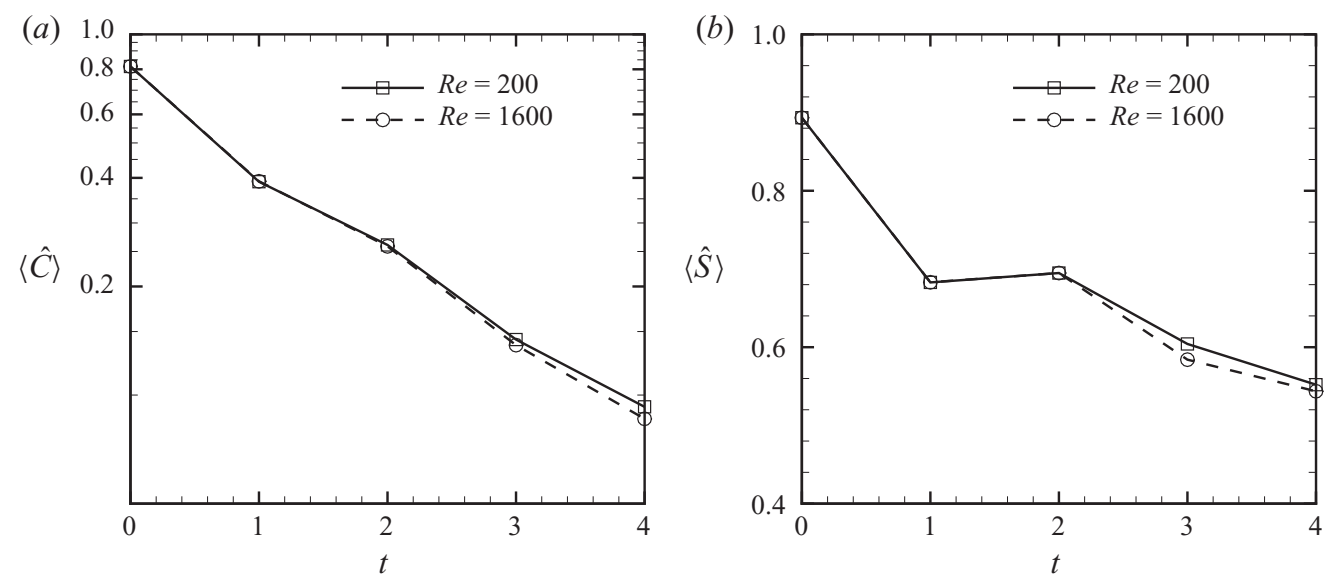

FIGURE 15. Averaged feature centres $\langle\hat{C}\rangle$ and $\langle\hat{S}\rangle$ at different times in the TG flow.

early stage are umbrella-like or parabolic. This observation is also visualized as the appearance of rolled-up structures at the edge of the vortex surface in figure 14(d).

To quantify these observations in the evolution, $\langle\hat{C}\rangle$ and $\langle\hat{S}\rangle$ are averaged over vortex surfaces at nine contour levels from 0.1 to 0.9 in the TG flow at $R e=1600$. As shown in figure $15(a)$, we can see that the averaged curvedness $\langle\hat{C}\rangle$ of vortex surfaces decreases exponentially with increasing time, which shows the strong flattening process of vortex surfaces corresponding to the growth of surface area, but in the sense of curvatures of surfaces. From figure $15(b)$, we find the averaged absolute value of the shape index $\langle\hat{S}\rangle$ appears to decrease with time and the value approaches 0.5 at $t=4$. Furthermore, lower $\langle\hat{C}\rangle$ and $\langle\hat{S}\rangle$ are obtained in the high-Re flow. This indicates that the vortex surfaces in the high-Re flow tend to be more nearly singular than those in the low- $R e$ flow, which may imply extreme, near-singular structures in the inviscid flow when $t \rightarrow t^{*}$.

\subsection{Relations between vortex-surface fields and vorticity fields}

As shown in figure 16, the geometry of vortex surfaces differs from that of the iso-surfaces of vorticity magnitude in the TG flow. In particular, in figure $16(b)$, the 'vorticity pancakes' represented by the high-vorticity regions, as shown in Brachet et al. (1992), appear to be generated between neighbouring vortex surfaces when they approach each other. The sheet-like structure of the high-vorticity region in simulations with other initial conditions (e.g. Pumir \& Siggia 1990 and Kerr 1993) could be induced by the similar approaching process of paired vortices. Referring to figure 10, vortex surfaces appear to reveal strong dynamics in the evolution of vortical structures, including stretching and subsequent roll-up. Since, for an inviscid flow, a vortex surface is topologically invariant, tracking the Lagrangian structure unfolds a continuous dynamic process. In contrast, the vorticity-magnitude iso-surface obeys no continuous dynamical form. Its instantaneous geometry thus results in many segmented structures with less implications for dynamics.

Compared with the TG flow, the vortex surface in the KP flow shows more interesting vortex dynamics in producing predominant large-scale spiral signatures. The evolution of eight vortex surfaces which lie in the $x-y$ plane with $-\pi / 2 \leqslant x, y \leqslant \pi / 2$ at $z=0$ is shown in figure 17 and the corresponding crosssections of vortex surfaces are shown in figure 18. At the initial time, eight 
(a)

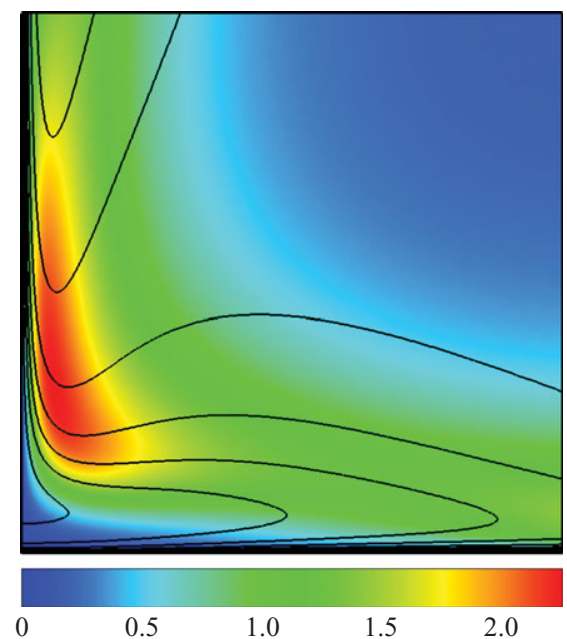

(b)

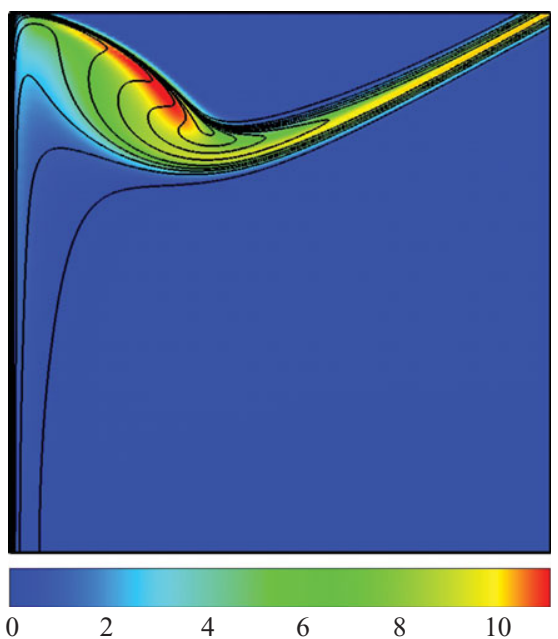

Figure 16. (Colour online) Relations between vortex surfaces and vorticity fields at $t=4$ in the TG flow at $R e=1600$. Vortex surface cross-sections are expressed by contour lines of $\phi$ and the magnitude of $|\omega|$ in shadings on the plane-cuts in the fundamental box. (a) $x-y$ plane-cut at $z=0$ and $(b) y-z$ plane-cut at $x=0$.

head-to-tail connected (without contact) vortex surfaces form a ring. The neighbouring vortex surfaces are not shown for clarity. We remark that, at a particular isocontour level, there are 192 vortex surfaces with certain symmetries in the box with $-\pi / 2 \leqslant x, y, z \leqslant 3 \pi / 2$. We can find two evolutionary geometries of vortex surfaces in the KP flow, which are, respectively, indicated in white and black in figure 17. The blob-like vortex surfaces are progressively distorted into two types of shell-like shapes. As shown in figure $17(d)$, each white vortex surface is wrapped by a black one and connected with another black one. The rolled-up structure will appear at the edge of vortex surfaces, as indicated in figure $18(d)$. From the iso-contour lines in figure 18 , we can see that the geometry of vortex surfaces is more regular than the vorticity contour lines shown in Boratav \& Pelz (1994) and better exemplify the vortex dynamics. All the vortex surfaces are wound in the clockwise direction on the $x-y$ plane-cut at $z=0$. In figure $18(d)$, multi-layered structures are generated at the joint of vortex surfaces at the later stages of our simulation.

The accumulation of vorticity may be related to the local geometry of vortex surfaces. Using a two-dimensional analogy, Constantin et al. (1994) found that rapid growth of vorticity appears to be induced at hyperbolic saddles of vortex lines. By solving the Euler-Lagrange equations for the associated variational problem, Lu \& Doering (2008) observed that vorticity is concentrated at the same place where vortex stretching occurs in a three-dimensional flow with the estimation of the maximum enstrophy growth rate. From figures $16(b)$ and 19, local hyperbolic or stretched shapes are also observed in the vicinity of high-vorticity regions in the present threedimensional flows. This suggests that highly localized vorticity intensification may be produced by the roll-up of vortex surfaces induced by non-parallel stretched vortex lines that comprise them. Thus, in terms of vortex-surface evolution, transition from the smooth initial flow condition to a flow state with almost singular structures, or even subsequent turbulent flow, can perhaps be understood in the following scenario. Initially, blob-like vortices are stretched and they approach each other. The 
(a)

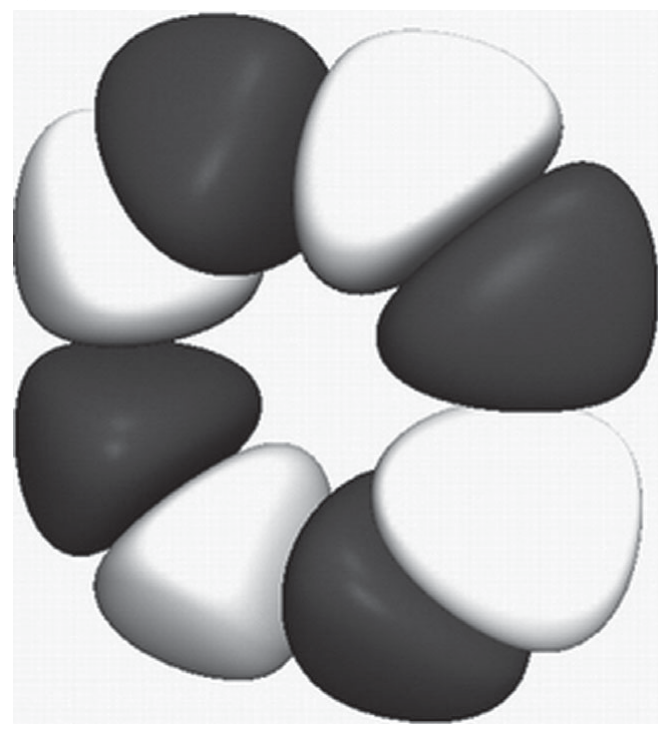

(c)

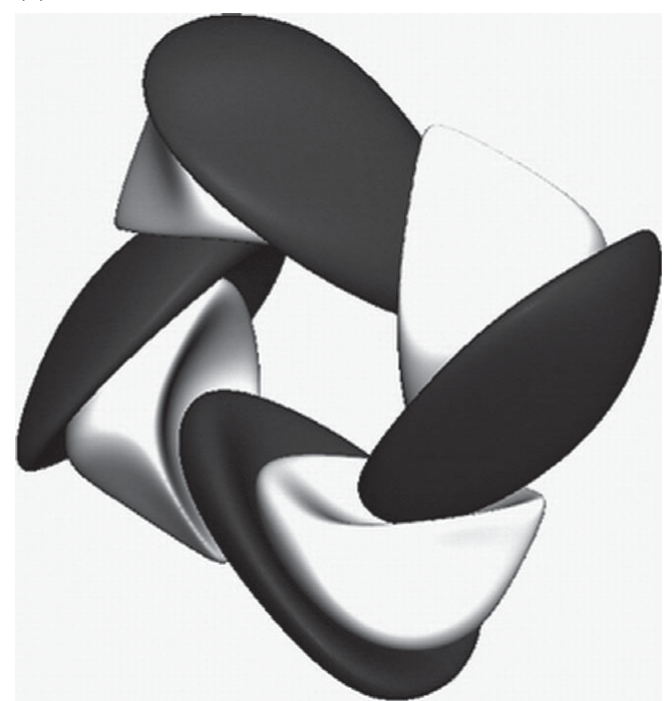

(b)

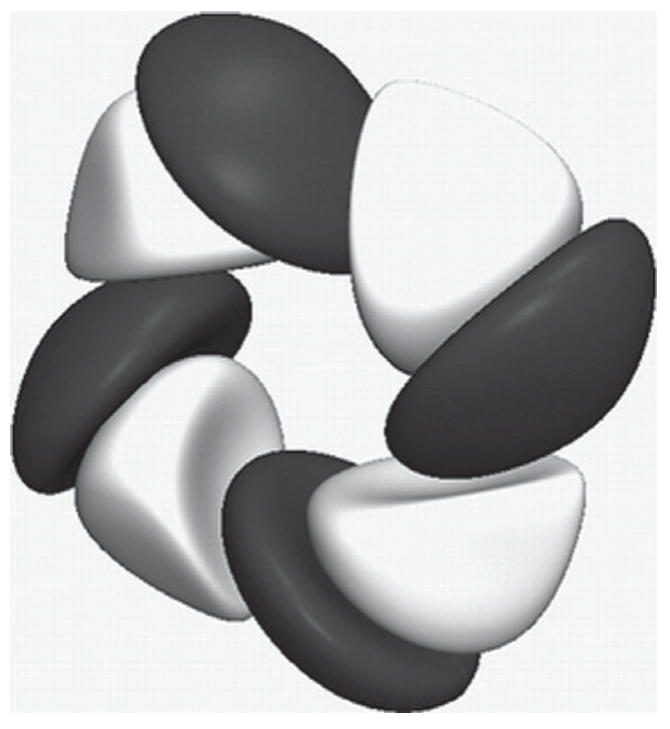

(d)

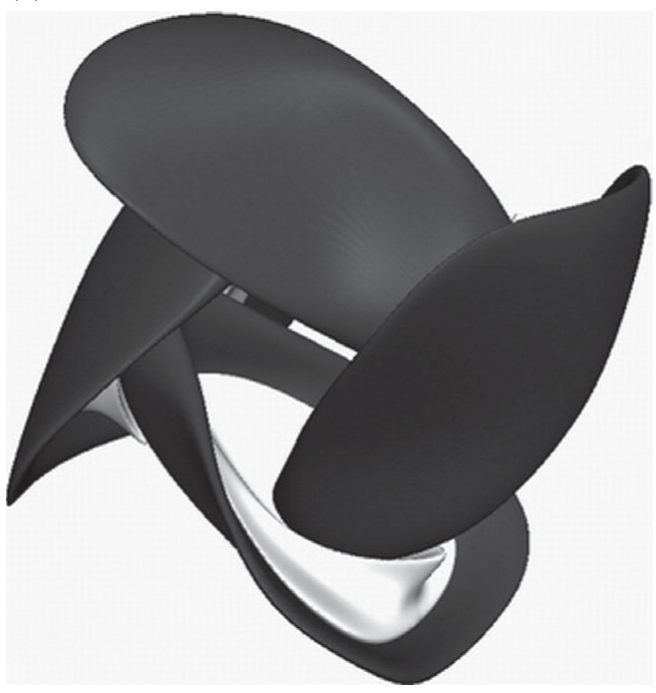

FIGURE 17. Vortex surfaces (iso-surfaces of $\hat{\phi}= \pm 0.015$ ) in the KP flow at $R e=1000$. These surfaces lie in the $x-y$ symmetry plane at $z=0$, where $-\pi / 2 \leqslant x, y \leqslant \pi / 2$. (a) $t=0,(b) t=0.25$, (c) $t=0.5$ and $(d) t=1$.

edges of stretched vortex surfaces are then gradually rolled up. Very high vorticity appears within the rolled-up structures at their edges and at the interface between neighbouring vortex surfaces. The hyperbolic structures near the high-vorticity regions might then become more unstable by viscosity-induced instabilities. This scenario is observed in the experiment of the head-on collision of two vortex rings (e.g. Lim \& Nickels 1993), in which the role of the dye is analogous to the vortex-surface scalar field used in the present study to identify the vortical structures in the Lagrangian sense. 
(a)

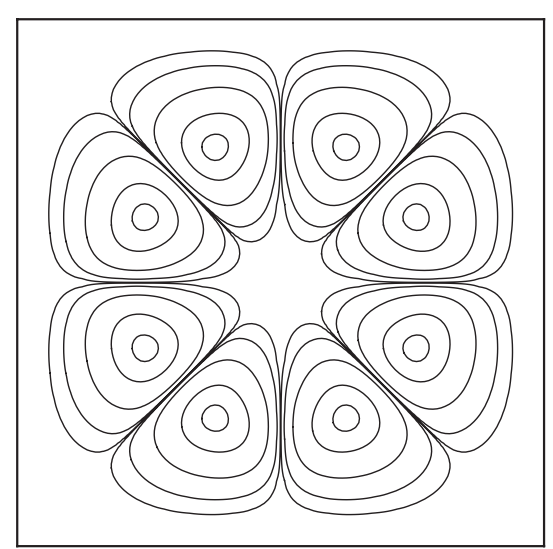

(c)

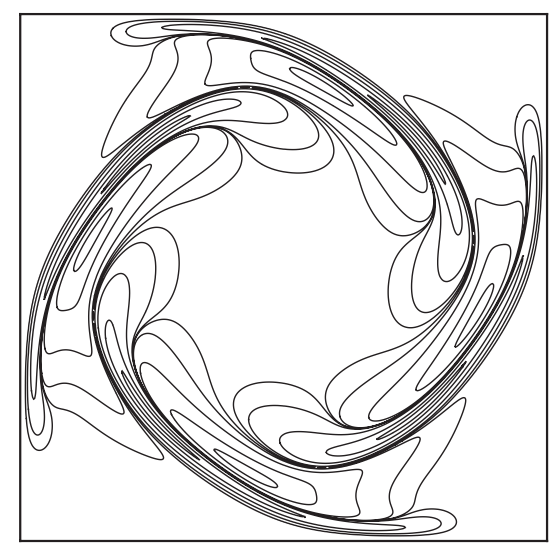

(b)

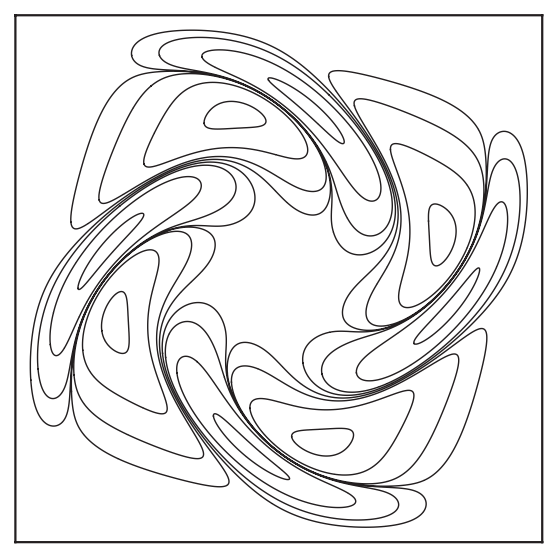

(d)

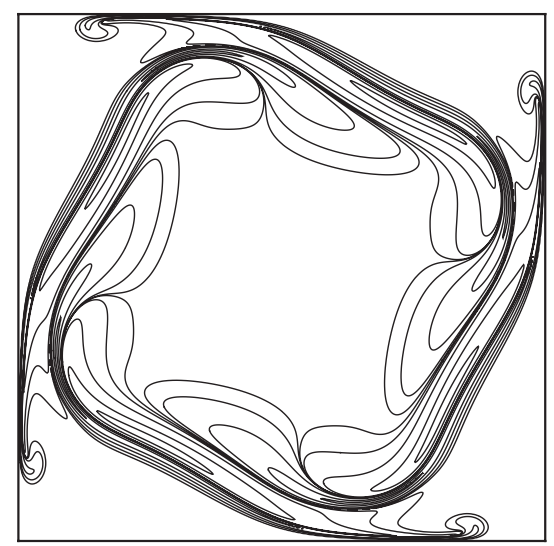

FigURE 18. Iso-contour lines of $\hat{\phi}=\{ \pm 0.001, \pm 0.01, \pm 0.1, \pm 0.5, \pm 0.9\}$ on the $x-y$ plane-cut at $z=0$ in the impermeable box for the KP flow at $R e=1000$. $(a) t=0,(b) t=0.5,(c) t=1$ and $(d) t=1.5$.

\section{Conclusions}

In this study, we have developed a systematic methodology for the construction of vortex-surface fields for both TG and KP initial velocity-vorticity fields. The spatial constraint on a three-dimensional scalar field $\phi$ is converted to a system of linear homogenous equations with Fourier expansions. In particular, although it appears that an exact and unique $\phi$ for the KP flow with a finite set of Fourier modes cannot be found, we can construct a least-squares solution through an optimization problem with the proposed basis functions that satisfy all KP symmetries. The somewhat elaborate scheme required to obtain vortex surfaces corresponding to the relatively simple KP initial vorticity field is surprising and unexpected. We remark that the methodology presented in $\S 2$ may not be applicable for every spatially periodic Eulerian vorticity field, for example the non-integrable ABC flow. The TG and KP flows are periodic and have relatively simple topology. All their vortex lines are closed and unknotted at the initial time. General three-dimensional periodic vorticity fields could exhibit such complex topologies that a $\phi$, even itself non-spatially periodic, which satisfies the constraint (2.3), may not in general exist. This appears to be an 
(a)

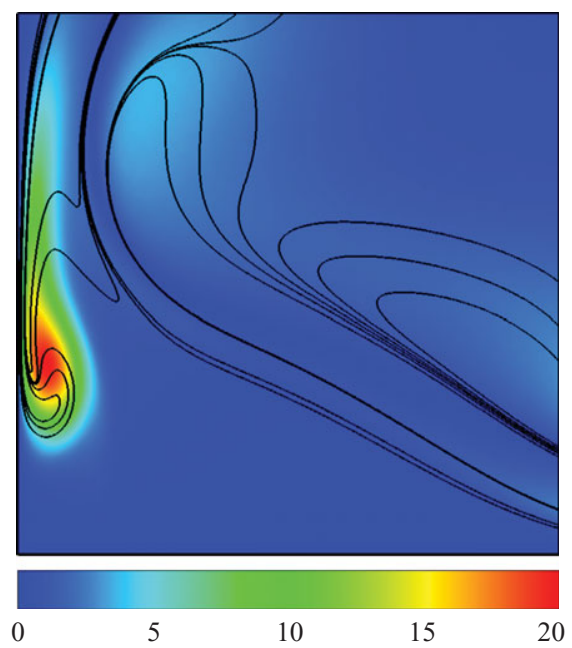

(b)

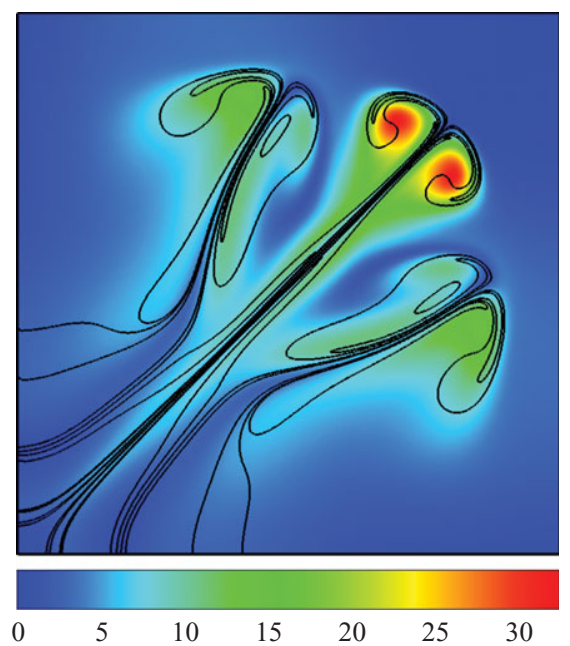

FIGURE 19. (Colour online) Relations between vortex surfaces and vorticity fields at $t=1.5$ in the KP flow at $R e=1000$. Vortex surface cross-sections are expressed by contour lines of $\phi$ and the magnitude of $|\omega|$ in shadings on the plane-cuts in the fundamental box. $(a) x-y$ plane-cut at $z=0$ and $(b) x-y$ plane-cut at $x=\pi / 2$.

open problem. In addition, the relations and differences between vortex-surface fields and the classical Clebsch representation are discussed.

The evolution equations of the vortex-surface field in both inviscid and viscous incompressible flows are derived in $\S 3$. In the inviscid case, with a well-defined initial vortex-surface field, the vortex-surface field is equivalent to the Lagrangian field and the non-uniqueness of the solution exists only up to relabelling. In the viscous case, solutions of equations describing a vortex-surface field, if they exist, are shown to be non-unique.

From our quantitative estimates, the relative error of vortex surfaces as Lagrangian surfaces is typically less than $5 \%$ in high-Reynolds-number TG and KP flows prior to times typical of a potential singularity time $t^{*}$. The error appears to be mainly associated with the topological constraint that prevents reconnection of Lagrangian structures, except possibly at singularities in a strictly Euler flow. This has the consequence that, for slightly viscous flows, vortex surfaces can be well approximated as Lagrangian structures prior to vortex reconnection. In temporal evolution, initial blob-like vortex surfaces are progressively stretched to sheet-like shapes so that neighbouring vortex surfaces approach each other. This flattening process of vortex surfaces is quantified by the growth rate of the surface area and the averaged curvedness $\langle\hat{C}\rangle$. The local topology of vortex surfaces is well represented by the averaged absolute value of the shape index $\langle\hat{S}\rangle$, which indicates that more local hyperbolic shapes on the surfaces appear near a potential $t^{*}$ than at the early stages of the evolution. We observed that local rolled-up shapes are predominantly located in the vicinity of high-vorticity regions, which might be related to the subsequent transition to turbulence. Furthermore, visualization of the evolution of vortex surfaces shows a continuous dynamic process of vortex roll-up with certain symmetries, which was not observed in visualizations of iso-surfaces of vorticity magnitude.

An interesting problem suggested by the present study is the development of general numerical methods for the identification and tracking of vortex surfaces in 
other flows, including viscous flows. In particular, the identification of vortex surfaces in vortex reconnection is important but challenging (Kida \& Takaoka 1994). Recent progress on the computation of invariant manifolds (see, e.g., Krauskopf et al. 2005 and Branicki \& Wiggins 2009) may shed light on the construction or identification of vortex surfaces. Another extension of the present study may address the relationship between the geometry of vortex surfaces and potential finite-time singularity in Euler flows (see Hou \& Li 2008). This, however, requires much higher resolution for both the Eulerian velocity field and the Lagrangian field than is employed presently. An adaptive mesh for $\phi$ in the Euler flow should be considered owing to the intermittently distributed nearly singular structures in vortex surfaces close to a potential $t^{*}$. Finally, the evolution of vortex surfaces may provide a reference for the development of vortex dynamics in a Lagrangian framework and new objective criteria for identifying vortical structures.

This work has been supported in part by the National Science Foundation under Grant DMS-0714050.

\section{Appendix A. Orthogonal basis functions with Kida-Pelz symmetries}

We propose an expansion of the vortex-surface field $\phi_{K P}$ based on the following fundamental functions, or linear combinations thereof, which satisfy the symmetries (Kida 1985) in the KP flow:

$$
\begin{aligned}
f_{0}(M, N, L)= & \left(\cos m_{1} x \cos m_{0} y-\cos m_{0} x \cos m_{1} y\right) \\
& \times\left(\cos n_{1} y \cos n_{0} z-\cos n_{0} y \cos n_{1} z\right) \\
& \times\left(\cos l_{1} z \cos l_{0} x-\cos l_{0} z \cos l_{1} x\right)
\end{aligned}
$$

where the wavenumber pairs,

$$
M=\left\{m_{0}, m_{1}\right\}, \quad N=\left\{n_{0}, n_{1}\right\}, \quad L=\left\{l_{0}, l_{1}\right\},
$$

are chosen by the following procedure.

(i) Each wavenumber pair $M, N$ or $L$ contains two different odd wavenumbers with $1 \leqslant m_{0}<m_{1}, 1 \leqslant n_{0}<n_{1}$ and $1 \leqslant l_{0}<l_{1}$. Let

$$
K_{\psi}=\max \left(m_{1}\right)=\max \left(n_{1}\right)=\max \left(l_{1}\right),
$$

and the maximum wavenumber $K_{F}=2 K_{\psi}$ in all the $f_{0}(M, N, L)$ considered for the approximation. The corresponding number of wavenumber pairs is

$$
N_{p r}=\left(K_{\psi}^{2}-1\right) / 8
$$

(ii) A list of wavenumber pairs obtained in the last step is built and sorted by the sum $\Sigma_{p}=2 m_{0}+m_{1}$ for each pair. Then each wavenumber pair is labelled by a pair index number $I_{p}$ from 1 to $N_{p r}$ by the sequence in the list. When a number of wavenumber pairs have the same $\Sigma_{p}$, the pair with a smaller $m_{0}$ has a smaller $I_{p}$. For example, a list of wavenumber pairs for $K_{\psi}=17$ is shown in table 3 . Here, we remark that the sequence in the list of wavenumber pairs may slightly affect the final solution quality and the convergence rate.

(iii) Each basis function $\psi_{i}$ is based on two types of combinations of the fundamental solution (A 1). The first type is

$$
\psi_{i}=f_{0}\left(M_{i}, N_{i}, L_{i}\right), \quad 1 \leqslant i \leqslant N_{p r}
$$




$\begin{array}{ccr}\text { Index number } I_{p} & \text { Wavenumber pair } & \Sigma_{p} \\ 1 & (1,3) & 5 \\ 2 & (1,5) & 7 \\ 3 & (1,7) & 9 \\ 4 & (1,9) & 11 \\ 5 & (3,5) & 11 \\ 6 & (1,11) & 13 \\ 7 & (3,7) & 13 \\ \vdots & \vdots & \vdots \\ 35 & (13,17) & 43 \\ 36 & (15,17) & 47\end{array}$

TABLE 3. List of wavenumber pairs $\left(K_{\psi}=17\right)$.

where the wavenumber pair is determined by its subscript corresponding to the pair index number $I_{p}$. The second type is

$$
\psi_{i}=f_{0}\left(M_{a}, N_{b}, L_{b}\right)+f_{0}\left(M_{b}, N_{a}, L_{b}\right)+f_{0}\left(M_{b}, N_{b}, L_{a}\right), \quad N_{p r}+1 \leqslant i \leqslant N_{\psi},
$$

with

$$
i=a N_{p r}-\frac{1}{2} a(a+1)+b, \quad 1 \leqslant a \leqslant N_{p r}-1, \quad a+1 \leqslant b \leqslant N_{p r},
$$

and the total number of the basis functions

$$
N_{\psi}=\frac{1}{2} N_{p r}\left(N_{p r}+1\right) \text {. }
$$

We find that both types of $\psi_{i}$ satisfy the reflectional symmetry about all three zero planes, and the rotational symmetry is expressed by the permutation symmetry:

$$
\psi_{i}(x, y, z)=\psi_{i}(z, x, y)=\psi_{i}(y, z, x) .
$$

In other words, the basis function $\psi_{i}$ is invariant under the action of the full octahedral symmetry group to match the same symmetry property in the KP flow (Pelz 2001).

Finally, orthogonal basis functions $\widetilde{\psi}_{i}$ are obtained from $\psi_{i}$ by the Gram-Schmidt orthogonalization,

$$
\widetilde{\psi}_{1}=\psi_{1} /\left\|\psi_{1}\right\|
$$

with

$$
\|f\|=\left(\int_{0}^{2 \pi} \int_{0}^{2 \pi} \int_{0}^{2 \pi} f \mathrm{~d} x \mathrm{~d} y \mathrm{~d} z\right)^{1 / 2},
$$

and then

$$
\left.\begin{array}{l}
\psi_{i}^{\prime}=\psi_{i}-\sum_{j=1}^{i-1}\left\langle\psi_{i}, \widetilde{\psi}_{j}\right\rangle \widetilde{\psi}_{j}, \quad i \geqslant 2, \\
\widetilde{\psi}_{i}=\psi_{i}^{\prime} /\left\|\psi_{i}\right\|,
\end{array}\right\}
$$

with

$$
\left\langle f_{1}, f_{2}\right\rangle=\left(\int_{0}^{2 \pi} \int_{0}^{2 \pi} \int_{0}^{2 \pi} f_{1} f_{2} \mathrm{~d} x \mathrm{~d} y \mathrm{~d} z\right)^{1 / 2}
$$




\section{Appendix B. Improvement on the smoothness of vortex-surface fields for the} Kida-Pelz initial field

In implementation of the optimization method in $\S 2.3$, we found that the iso-surface of $\phi_{K P}$ from (2.23) at very small contour levels may exhibit non-smoothness. This could be a numerical consequence of non-uniqueness of solutions to (2.3) or perhaps to instability in solving the large-scale linear system as $N_{\psi}$ is large. For $N_{\psi}=666$, the matrix $\boldsymbol{A}$ with size 53536 by 665 is sparse. Hence, an additional constraint on $\left\|\boldsymbol{\Psi}^{\prime}\right\|_{2}$ was applied in this least-squares problem, which will both improve the smoothness of the approximate solution at very small contour levels and at the same time provide uniqueness at the numerical level. This results in a quadratic optimization problem,

$$
\min _{\boldsymbol{\Psi}^{\prime}}\left\|\boldsymbol{A} \boldsymbol{\Psi}^{\prime}-\boldsymbol{b}\right\|_{2}^{2}+\lambda\left\|\boldsymbol{\Psi}^{\prime}\right\|_{2}^{2},
$$

where the ridge parameter $\lambda=3.75 \times 10^{-8}$ is introduced to obtain the local minimum value of $\left|\nabla \phi_{K P}\right|_{\max }$. This ridge regression problem has an analytical solution (see Golub \& Van Loan 1996)

$$
\boldsymbol{\Psi}^{\prime}=\left(\boldsymbol{A}^{\mathrm{T}} \boldsymbol{A}+\lambda \boldsymbol{I}\right)^{-1} \boldsymbol{A}^{\mathrm{T}} \boldsymbol{b} .
$$

To solve this quadratic optimization problem numerically by the highly efficient driver routine with the QR method in LAPACK (Anderson et al. 1999), (B 1) is converted to a linear least-squares problem:

$$
\min _{\boldsymbol{\Psi}^{\prime}}\left\|\left(\begin{array}{c}
\boldsymbol{A} \\
\sqrt{\lambda} \boldsymbol{l}
\end{array}\right) \boldsymbol{\Psi}^{\prime}-\left(\begin{array}{c}
\boldsymbol{b} \\
0
\end{array}\right)\right\|_{2}^{2} .
$$

\section{Appendix C. Computation of vortex-surface fields in an incompressible viscous flow}

From $\S 3$, for $\phi_{v}$ to evolve as the vortex-surface field in a viscous flow satisfying (3.4), we must solve (3.13), (3.14) and (4.1) simultaneously. Here, (3.14) is a special case of the generalized first-order PDE:

$$
\omega_{j}(\boldsymbol{x}) \frac{\partial \mathscr{L}(\boldsymbol{x})}{\partial x_{j}}+B(\boldsymbol{x}) \mathscr{L}(\boldsymbol{x})=f(\boldsymbol{x}) .
$$

With the finite Fourier expansion

$$
\mathscr{L}(\boldsymbol{x})=\sum_{\boldsymbol{k}} \hat{\mathscr{L}}(\boldsymbol{k}) \mathrm{e}^{\mathrm{i} k \cdot \boldsymbol{x}},
$$

(C 1) could be computed by a spectral method (see Mingyu et al. 1997). By multiplying $\mathrm{e}^{-\mathrm{i} k^{\prime} \cdot x}$ on both sides of (C1), we can obtain the $k^{\prime}$ th equation for a linear system,

$$
\sum_{\boldsymbol{k}}\left(\mathrm{i} k_{j} \hat{\omega}_{j}\left(\boldsymbol{k}^{\prime}-\boldsymbol{k}\right)+\hat{B}\left(\boldsymbol{k}^{\prime}-\boldsymbol{k}\right)\right) \hat{\mathscr{L}}(\boldsymbol{k})=\hat{f}\left(\boldsymbol{k}^{\prime}\right),
$$

where

$$
\begin{aligned}
\hat{\omega}_{j}\left(\boldsymbol{k}^{\prime}-\boldsymbol{k}\right) & =\frac{1}{(2 \pi)^{3}} \int \omega_{j}(\boldsymbol{x}) \mathrm{e}^{-\mathrm{i}\left(\boldsymbol{k}^{\prime}-\boldsymbol{k}\right) \cdot \boldsymbol{x}} \mathrm{d} \boldsymbol{x}, \\
\hat{B}\left(\boldsymbol{k}^{\prime}-\boldsymbol{k}\right) & =\frac{1}{(2 \pi)^{3}} \int B(\boldsymbol{x}) \mathrm{e}^{-\mathrm{i}\left(\boldsymbol{k}^{\prime}-\boldsymbol{k}\right) \cdot \boldsymbol{x}} \mathrm{d} \boldsymbol{x}, \\
\hat{f}\left(\boldsymbol{k}^{\prime}\right) & =\frac{1}{(2 \pi)^{3}} \int f(\boldsymbol{x}) \mathrm{e}^{-\mathrm{i} \boldsymbol{k}^{\prime} \cdot \boldsymbol{x}} \mathrm{d} \boldsymbol{x} .
\end{aligned}
$$


(a)

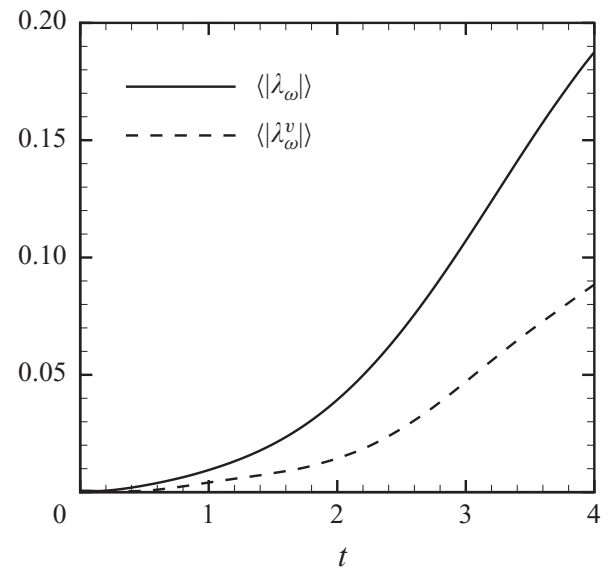

(b)

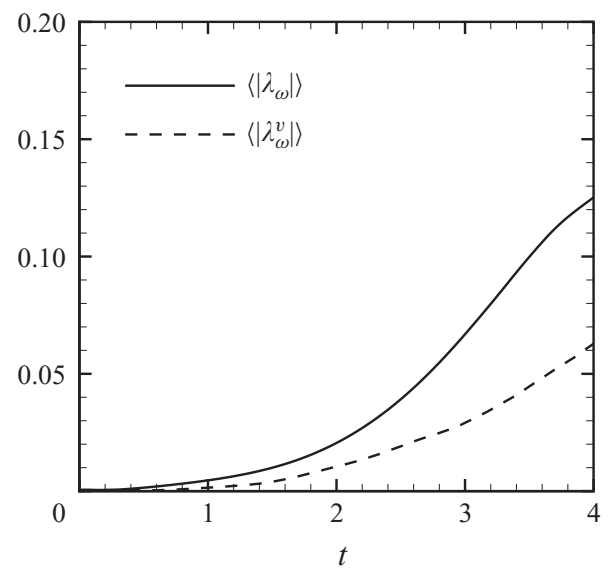

FIGURE 20. Temporal evolution of the volume-averaged $\left|\lambda_{\omega}\right|$ for Lagrangian simulations and $\left|\lambda_{\omega}^{v}\right|$ for vortex-surface simulations in the TG viscous flow at low Reynolds numbers. (a) $R e=25$ and (b) $R e=50$.

The PDE system (C1) is said to be dissipative if the following condition holds (Mingyu et al. 1997):

$$
\left\langle\left(B-\frac{1}{2}(\nabla \cdot \omega)\right) \boldsymbol{\eta}, \boldsymbol{\eta}\right\rangle>0, \quad \forall \boldsymbol{\eta} \in \mathbb{R}^{n} .
$$

Then (C 1) and the discrete system (C 3) have a unique solution. For (3.14), $f=-\nabla \phi$. $\nabla^{2} \omega, B=0, \nabla \cdot \omega=0$, and the condition (C7) for existence and uniqueness is not satisfied. We have nonetheless numerically demonstrated the existence of solutions to $(\mathrm{C} 1)$ with $B=f=0$ defining a vortex-surface field for the KP vorticity (2.15) by using an optimization formulation in $\S 2.3$.

Some explorative solutions have been performed for the vortex-surface field in the TG flow. The Navier-Stokes equations (4.1) were solved by the pseudo-spectral method, using the TG initial conditions (2.9) with $\theta=0$ and at Reynolds numbers $R e=25$ and $R e=50$ on the grids $64^{3}$. For each simulation, two separate scalar simulations were performed, both with initial scalar fields given by the TG vortexsurface field (2.12). In the Lagrangian simulation, (3.1) was calculated as a Lagrangian field $\phi$ using the backward-particle-tracking method, as described in $\S 4.2$. For this case, $\phi=\phi_{v}$ at $t=0$ but does not remain a vortex-surface field. In the vortex-surface simulation, (3.13) and (3.14) were solved for $\phi_{v}$ and $\mathscr{L}$. Since (3.13) contains a source term, the backward-particle-tracking method cannot be utilized. Instead, this equation was solved by a finite-difference method where the convection term was treated using a fifth-order weighted essentially non-oscillatory scheme (Jiang \& Shu 1996). The spectral method (see (C 3)) was utilized to solve (3.14). This produces a linear system

$$
\boldsymbol{A} \boldsymbol{L}=\boldsymbol{b},
$$

where $\boldsymbol{L}$ is the $1 \times n$ vector of Fourier coefficients for $\mathscr{L}$ and $n$ is determined by the number of effective Fourier modes after dealiasing. It is noted that considering the TG symmetries (Brachet et al. 1983), the Fourier representation for $\mathscr{L}$ in the TG flow could be

$$
\mathscr{L}(\boldsymbol{x})=\sum_{k_{1}} \sum_{k_{2}} \sum_{k_{3}} \hat{\mathscr{L}}\left(k_{1}, k_{2}, k_{3}\right) \cos k_{1} x \cos k_{2} y \cos k_{3} z
$$

and $\hat{\mathscr{L}}$ vanishes unless $k_{1}, k_{2}, k_{3}$ are either all even or all odd integers. 
Owing to non-uniqueness of (3.14), the $n \times n$ matrix $\boldsymbol{A}$ becomes rank-deficient and ill-conditioned. Further conditions on solutions of (3.14) are therefore required. Integrating the difference between (3.13) and (3.1) over a $(2 \pi)^{3}$ domain in physical space with periodic boundary conditions on all quantities gives

$$
\int_{0}^{2 \pi} \int_{0}^{2 \pi} \int_{0}^{2 \pi}\left(\frac{\mathrm{D} \phi_{v}}{\mathrm{D} t}-\frac{\mathrm{D} \phi}{\mathrm{D} t}\right)^{2} \mathrm{~d} x \mathrm{~d} y \mathrm{~d} z=v^{2} \int_{0}^{2 \pi} \int_{0}^{2 \pi} \int_{0}^{2 \pi} \mathscr{L}^{2} \mathrm{~d} x \mathrm{~d} y \mathrm{~d} z .
$$

For $v=0$, the volume integral of $\mathscr{L}^{2}$ is zero in the Lagrangian case. A reasonable choice for $v \neq 0$ could then be minimizing the integral of $|\mathscr{L}|$. With the Fourier method, the minimum norm solution was obtained which minimizes $\|\boldsymbol{L}\|_{2}$ in (C 8) using the singular value decomposition in LAPACK (Anderson et al. 1999). Here, $\boldsymbol{A}=\boldsymbol{U} \boldsymbol{\Sigma} \boldsymbol{V}^{\mathrm{T}}$, where $\boldsymbol{U}$ and $\boldsymbol{V}$ are $n \times n$ satisfying $\boldsymbol{U}^{\mathrm{T}} \boldsymbol{U}=\boldsymbol{I}$ and $\boldsymbol{V}^{\mathrm{T}} \boldsymbol{V}=\boldsymbol{I}$, respectively, and $\boldsymbol{\Sigma}=\operatorname{diag}\left(\sigma_{1}, \ldots, \sigma_{n}\right)$, where singular values $\sigma_{1} \geqslant \cdots \geqslant \sigma_{n} \geqslant 0$. In the implementation, singular values $\sigma_{i} \leqslant r_{\sigma} \sigma_{1}$ are treated as zero, where $r_{\sigma}$ is a small number.

The volume-averaged $\left|\lambda_{\omega}\right|$ for the Lagrangian simulation and $\left|\lambda_{\omega}^{v}\right|$ for the vortexsurface simulation are plotted in figure 20, where $\lambda_{\omega}^{v}$ is the cosine of the angle between $\omega$ and $\nabla \phi_{v}$. It may be seen that $\left\langle\left|\lambda_{\omega}^{v}\right|\right\rangle$ in the vortex-surface simulation is substantially less than $\left\langle\left|\lambda_{\omega}\right|\right\rangle$ in the Lagrangian simulation, but that this is not sufficiently small. Visual inspection of iso-surfaces of the scalar fields (not shown), however, indicate that the attachment of vortex lines to the iso-surface is substantially superior for the vortex-surface simulations than for the Lagrangian simulations. More investigations on the evolution of vortex-surface fields in a viscous flow will be left for future research.

\section{REFERENCES}

Anderson, E., Bai, Z., Bischof, C., Blackford, S., Demmel, J., Dongarra, J., Du Croz, J., Greenbaum, A., Hammarling, S., McKenney, A. \& Sorensen, D. 1999 LaPACK Users' Guide, 3rd edn. Society for Industrial and Applied Mathematics.

Ashurst, W. T., Kerstein, A. R., Kerr, R. M. \& Gibson, C. H. 1987 Alignment of vorticity and scalar gradient with strain rate in simulated Navier-Stokes turbulence. Phys. Fluids 30, 2343-2353.

Bermejo-Moreno, I. \& Pullin, D. I. 2008 On the non-local geometry of turbulence. J. Fluid Mech. 603, 101-135.

Boratav, O. N. \& Pelz, R. B. 1994 Direct numerical simulation of transition to turbulence from a high-symmetry initial condition. Phys. Fluids 6, 2757-2784.

Brachet, M. E., Meiron, D. I., Orszag, S. A., Nickel, B. G., Morf, R. H. \& Frisch, U. 1983 Small-scale structure of the Taylor-Green vortex. J. Fluid Mech. 130, 411-452.

Brachet, M. E., Meneguzzi, M., Vincent, A., Politano, H. \& Sulem, P. L. 1992 Numerical evidence of smooth self-similar dynamics and possibility of subsequent collapse for three-dimensional ideal flows. Phys. Fluids A 4, 2845-2854.

Branicki, M. \& Wiggins, S. 2009 An adaptive method for computing invariant manifolds in non-autonomous, three-dimensional dynamical systems. Physica D 238, 1625-1657.

Cartes, C., Bustamante, M. D. \& Brachet, M. E. 2007 Generalized Eulerian-Lagrangian description of Navier-Stokes dynamics. Phys. Fluids 19, 077101.

Chakraborty, P., Balachandar, S. \& Adrian, R. J. 2005 On the relationships between local vortex identification schemes. J. Fluid Mech. 535, 189-214.

Chong, M. S., Perry, A. E. \& Cantwell, B. J. 1990 A general classification of three-dimensional flow fields. Phys. Fluids A 2, 765-777.

ClebsCH, A. 1859 Ueber die Integration der hydrodynamischen Gleichungen. J. Reine Angew. Math. 56, $1-10$.

Constantin, P. 2001 An Eulerian-Lagrangian approach to the Navier-Stokes equations. Commun. Math. Phys. 216, 663-686. 
Constantin, P., Majda, A. J. \& Tabak, E. 1994 Formation of strong fronts in the 2-D quasigeostrophic thermal active scalar. Nonlinearity 7, 1495-1533.

Dieci, L., Lorenz, J. \& Russell, R. D. 1991 Numerical calculation of invariant tori. SIAM J. Sci. Stat. Comput. 12, 607-647.

Dombre, T., Frisch, U., Greene, J. M., Henon, M., Mehr, A. \& Soward, A. M. 1986 Chaotic streamlines in the ABC flows. J. Fluid Mech. 167, 353-391.

Ertel, H. 1942 Ein neuer hydrodynamischer Wirbelsatz. Meteorol Z. 59, 271-281.

Golub, G. H. \& Van LoAn, C. F. 1996 Matrix Computations, 3rd edn. Johns Hopkins University Press.

GoTo, S. \& KIDA, S. 2007 Reynolds-number dependence of line and surface stretching in turbulence: folding effects. J. Fluid Mech. 586, 59-81.

Haller, G. 2005 An objective definition of a vortex. J. Fluid Mech. 525, 1-26.

Helmholtz, H. 1858 Über Integrale der hydrodynamischen Gleichungen welche den Wirbelbewegungen ensprechen. J. Reine Angew. Math. 55, 25-55.

Hou, T. Y. \& LI, R. 2008 Blowup or no blowup? The interplay between theory and numerics. Physica D 237, 1937-1944.

Hunt, J. C. R., Wray, A. A. \& Moin, P. 1988 Eddies, stream, and convergence zones in turbulent flows. Center for Turbulence Research Rep. CTR-S88, pp. 193-208.

JeOng, J. \& Hussain, F. 1995 On the identification of a vortex. J. Fluid Mech. 285, 69-94.

JiANG, G. S. \& SHU, C. W. 1996 Efficient implementation of weighted ENO schemes. J. Comput. Phys. 126, 202-228.

KERR, R. M. 1993 Evidence for a singularity of the three-dimensional, incompressible Euler equations. Phys. Fluids A 5, 1725-1746.

KIDA, S. 1985 Three-dimensional periodic flows with high-symmetry. J. Phys. Soc. Japan 54, 2132 2136

Kida, S. \& Takaoka, M. 1994 Vortex reconnection. Annu. Rev. Fluid Mech. 26, 169-189.

KoEnderink, J. J. \& VAN Doorn, A. J. 1992 Surface shape and curvature scales. Image Vision Comput. 10, 557-564.

Krauskopf, B., Osinga, H. M., Doedel, E. J., Henderson, M. E., Guckenheimer, J., Vladimirsky, A., Dellnitz, M. \& Junge, O. 2005 A survey of methods for computing (un)stable manifold of vector fields. Intl J. Bifurcation Chaos 15, 763-791.

Lamb, H. 1932 Hydrodynamics, 6th edn. Cambridge University Press.

LiM, T. T. \& NicKELS, T. B. 1993 Instability and reconnection in the head-on collision of two vortex rings. Nature 357, 225-227.

LiNDSAY, K. \& KRASNY, R. 2001 A particle method and adaptive treecode for vortex sheet motion in three-dimensional flow. J. Comput. Phys. 172, 879-907.

Lu, L. \& Doering, C. R. 2008 Limits on enstrophy growth for solutions of the three-dimensional Navier-Stokes equations. Indiana Univ. Math. J. 57, 2693-2727.

Majda, A. J. \& Bertozzi, A. L. 2001 Vorticity and Incompressible Flow. Cambridge University Press.

Mingyu, H., KüPper, T. \& Masbaum, N. 1997 Computation of invariant tori by the Fourier methods. SIAM J. Sci. Comput. 18, 918-942.

Nore, C., Abid, M. \& Brachet, M. E. 1997 Decaying Kolmogorov turbulence in a model of superflow. Phys. Fluids 9, 2644-2669.

OhKitani, K. 2008 A geometrical study of 3D incompressible Euler flows with Clebsch potentials: a long-lived Euler flow and its power-law energy spectrum. Physica D 237, 2020-2027.

Ohkitani, K. \& Constantin, P. 2003 Numerical study of the Eulerian-Lagrangian formulation of the Navier-Stokes equations. Phys. Fluids 15, 3251-3254.

Pelz, R. B. 2001 Symmetry and the hydrodynamic blow-up problem. J. Fluid Mech. 444, 299-320.

Pullin, D. I. \& Saffman, P. G. 1998 Vortex dynamics in turbulence. Annu. Rev. Fluid Mech. 30, $31-51$.

Pumir, A., Shraiman, B. I. \& Siggia, E. D. 1992 Vortex morphology and Kelvin's theorem. Phys. Rev. A 45, R5351-R5354.

Pumir, A. \& Siggia, E. 1990 Collapsing solutions to the 3-D Euler equations. Phys. Fluids A 2, $220-241$. 
Ruetsch, G. R. \& Maxey, M. R. 1992 The evolution of small-scale structures in homogeneous isotropic turbulence. Phys. Fluids A 4, 2747-2760.

Russo, G. \& Smereka, P. 1999 Impulse formulation of the Euler equations: general properties and numerical methods. J. Fluid Mech. 391, 189-209.

Saffman, P. G. 1992 Vortex Dynamics. Cambridge University Press.

Salmon, R. 1988 Hamiltonian fluid mechanics. Annu. Rev. Fluid Mech. 20, 225-256.

TAYLOR, G. I. \& GreEn, A. E. 1937 Mechanism of the production of small eddies from large ones. Proc. R. Soc. Lond. A 158, 499-521.

Wolfram ResEARCh, InC. 2008 Mathematica. v7.0.

YAng, Y., Pullin, D. I. \& Bermejo-Moreno, I. 2010 Multi-scale geometric analysis of Lagrangian structures in isotropic turbulence. J. Fluid Mech. 654, 233-270. 